\title{
Spatialisation et Analyse du Risque d'Inondation dans le Bassin versant de la Sebkha de l'Ariana (Tunisie Nord-orientale)
}

Walid CHOUARI*

\begin{abstract}
RÉSUMÉ
Le bassin versant de la sebkha de l'Ariana est topographiquement très contrasté avec une prédominance des formations imperméables argileuses et des terres hydromorphes des rives de la sebkha. Ce bassin versant a connu, depuis les années 1970, une extension urbaine spectaculaire. L'axe urbain Nord-est de l'agglomération tunisoise s'est étendu considérablement au détriment des terres marginales des rives de la sebkha sans un aménagement préalable.

Le problème de ce bassin versant réside dans le fait qu'à la moindre pluie importante, les eaux de ruissellement en provenance des reliefs environnants se rassemblent au niveau des voies de circulations fréquemment surélevées par rapport aux terrains qui les bordent. Puis les eaux s'épandent à l'aval dans une plaine à relief plat où l'évacuation des eaux vers la sebkha s'effectue difficilement en l'absence du réseau secondaire de drainage et de collecteurs principaux d'eaux pluviales de capacité suffisante. À ces phénomènes s'ajoutent des perturbations du bilan hydrologique de la zone avec l'augmentation de l'imperméabilisation du sol et l'accélération du processus de ruissellement impliquant, par conséquent, des augmentations des volumes de ruissellement au détriment des volumes infiltrés et donc des débits de pointe et du transport solide qui ne font qu'aggraver la situation.

Cette étude propose ainsi une méthodologie de cartographie des risques d'inondations centrée sur l'exposition de la vulnérabilité des enjeux aux aléas considérés. Le résultat de cette étude est une carte des risques d'inondations en trois niveaux dont l'intensité de la couleur exprime la valeur du risque. Les zones soumises à un risque fort couvrent une superficie de l'ordre de $40 \%$ du bassin versant et concernent surtout la voirie et les zones à densités urbaines moyennes à fortes soumises à des chasses d'eau au piémont de Jbel Nahli ou à une remontée du toit de la nappe phréatique sur les rives de la sebkha. L'étude s'inscrit dans un effort de recherche déjà entrepris depuis une quinzaine d'années et se distingue par l'utilisation de données multisources et multidates, notamment des photographies aériennes et des extraits de Google Earth. [Bul. Soc. Géog. d’Égypte, 2016, 89: 123-154]
\end{abstract}

Mots clés: sebkha de l'Ariana, inondation, cartographie, vulnérabilité, risque.

* Faculté des Lettres et Sciences Humaines, Université de SFAX, Tunis. 


\section{ABSTRACT}

The Ariana Sabkha basin is characterized by its diversity of landforms and dominance of impermeable clay formations, which cause a waterlogged land on its banks. Since the 1970's, the basin area faced a huge unplanned urban extension, due to migration. Large unplanned urban areas extended remarkably Northeastern of Tunis along the stream banks.

The problem of the watershed is that when it is exposed to significant rain, streams from the nearby slopes gather causing an overland flow, which spreads over roads along the level low land. Flood are caused by streams spreading towards the flat level areas since it is difficult to be drained, particularly in the absence of sufficient drainage. In addition, changes in land use causes soil physical properties degradation and great erosion that result in disturbing the hydrological balance, and accelerating overland flow, flooding, and sedimentation.

This study proposes a methodology of using multi-data sources for flood risks mapping, visualization and modeling on the exposure of the vulnerability of the land use. The methodology results in various flood risk maps. The zones subject to high risk cover about $40 \%$ of the basin area and concern the roadways and the zones with average and high urban densities. These zones are subject to flushing in piedmont of the mountain of Nahli or to an elevation of the level of the underground water. The study falls under a fifteen-year research effort and presents originality with the use of several multi-sources and multi-dates data, in particular, of the air photographs and the extracts of Google Earth.

Keywords: Ariana Sabkha, flood, cartography, vulnerability, risk.

\section{Introduction}

La croissance démesurée et non contrôlée de l'urbanisation dans le bassin versant de la sebkha de l'Ariana a posé des lourds problèmes à des terres qui sont, pour la plupart, non viables et impropres à l'aménagement. L'absence de mesures d'accompagnement pour la mise en place d'infrastructures de base afin de garantir un cadre de vie sain, a favorisé la dégradation des conditions environnementales. Et, l'axe urbain nord-orientale de l'agglomération tunisoise s'est, depuis les années 1970, étendu aux dépens des terres marécageuses et inondables des rives de la sebkha.

Les petits cours d'eau qui descendent des reliefs environnants, sont très actifs à l'occasion des phénomènes pluviométriques importants et dont l'écoulement se fait à fleur de sol dans la plaine. Les sections des oueds se trouvent envahies par les constructions et souvent remplacées en aval par des canaux de drainage plus ou moins fiables. Face à cette situation, des risques d'inondations lors des pluies importantes provoquent sur les rives de la sebkha une remontée de la nappe d'eau subaffleurante. Des zones proches des rives est de la sebkha sont aménagées, se trouvent à un niveau topographique 
inférieur à celui de la mer et sont soumises à des risques potentiels d'une submersion par les eaux du plan d'eau.

Ainsi, dans l'objectif de comprendre davantage le risque d'inondation qui menace l'homme dans ce bassin versant, cette étude propose ainsi une méthodologie de cartographie des risques d'inondation centrée sur l'exposition de la vulnérabilité des enjeux aux aléas considérés.

\section{I) Un bassin versant vulnérable aux inondations}

\section{La vulnérabilité physique du bassin versant}

Afin d'étudier la topographie locale du bassin versant nous avons réalisé un Modèle Numérique de Terrain à base de $5 \mathrm{~m}$. Le travail cartographique nous a permis de distinguer trois niveaux topographiques étagés. La première zone située entre la ligne de crête et la courbe de niveau $55 \mathrm{~m}$. Cette zone se caractérise par une pente forte à très forte avec des valeurs qui varient entre 10 et $25 \%$ (Fig. 1 ).

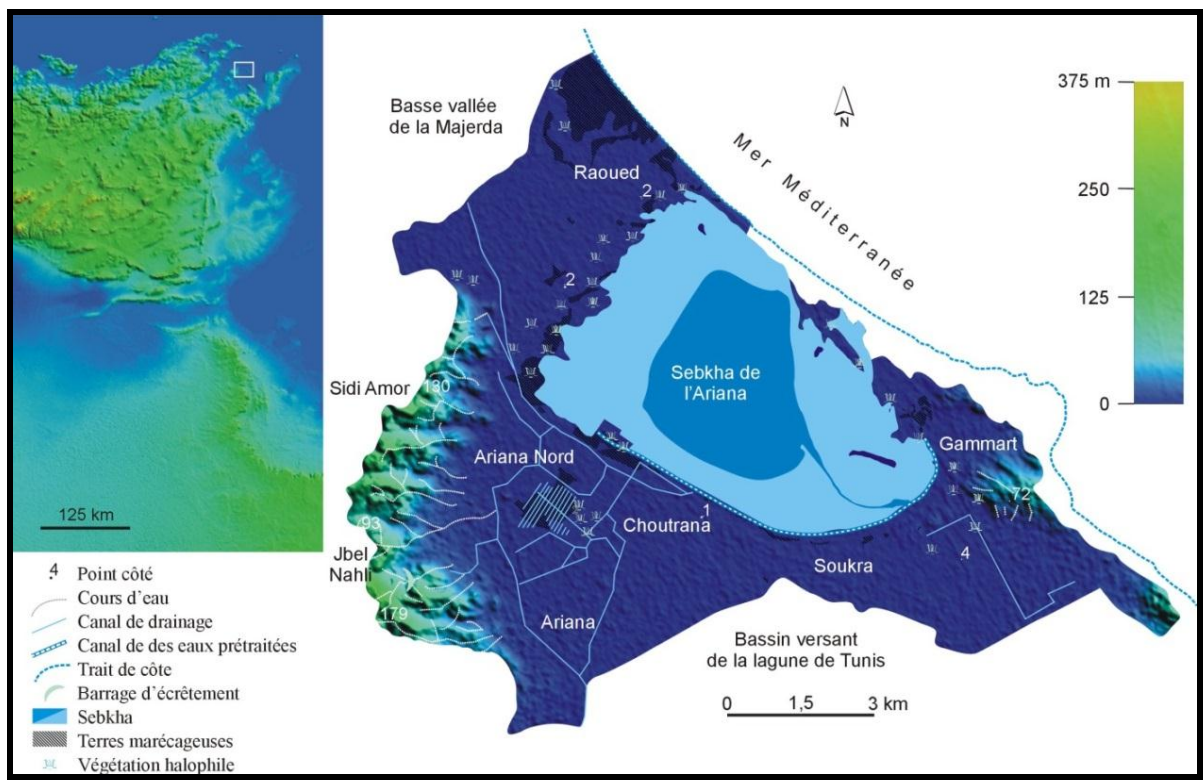

Fig. (1): Localisation du bassin versant de la sebkha de l'Ariana en Tunisie (gauche), et détails de sa topographie et de son hydrologie (droite).

Sources: Une image ASTER (données d'altitude à couverture globale, résolution spatiale $=20 \mathrm{~m}$ ) et des cartes topographiques type 1982 au 1/25000 (feuille $\mathrm{n}^{\circ} \mathrm{XIII}$ «NE et SE» et feuille $\mathrm{n}^{\circ} \mathrm{XIV} \ll \mathrm{NO}$ et $\left.\mathrm{SO} »\right)$. 
La deuxième zone située entre la courbe de niveau 55 et celle de $30 \mathrm{~m}$. Elle est étalée du Nord vers le Sud et d'une largeur ne dépassant pas quelques centaines de mètres. Elle correspond, en fait, au piedmont du Jbel et assure un passage graduel et parfois brutal entre la zone accidentée et la basse plaine. Elle est caractérisée par une pente globale dirigée du Sud-Ouest vers le Nord-Est avec des valeurs moyennes à faibles, généralement entre 2,5 et $10 \%$ (Fig. 2).

La troisième zone est limitée à l'amont par la courbe de niveau $30 \mathrm{~m}$ et en aval par les rives est de la sebkha de l'Ariana situées aux environs d'un mètre d'altitude. Elle est relativement plane, puisque la pente ne dépasse pas $2,5 \%$. Dans la majeure partie de la zone et surtout en aval de la RV543, la pente est presque nulle (Fig. 2).

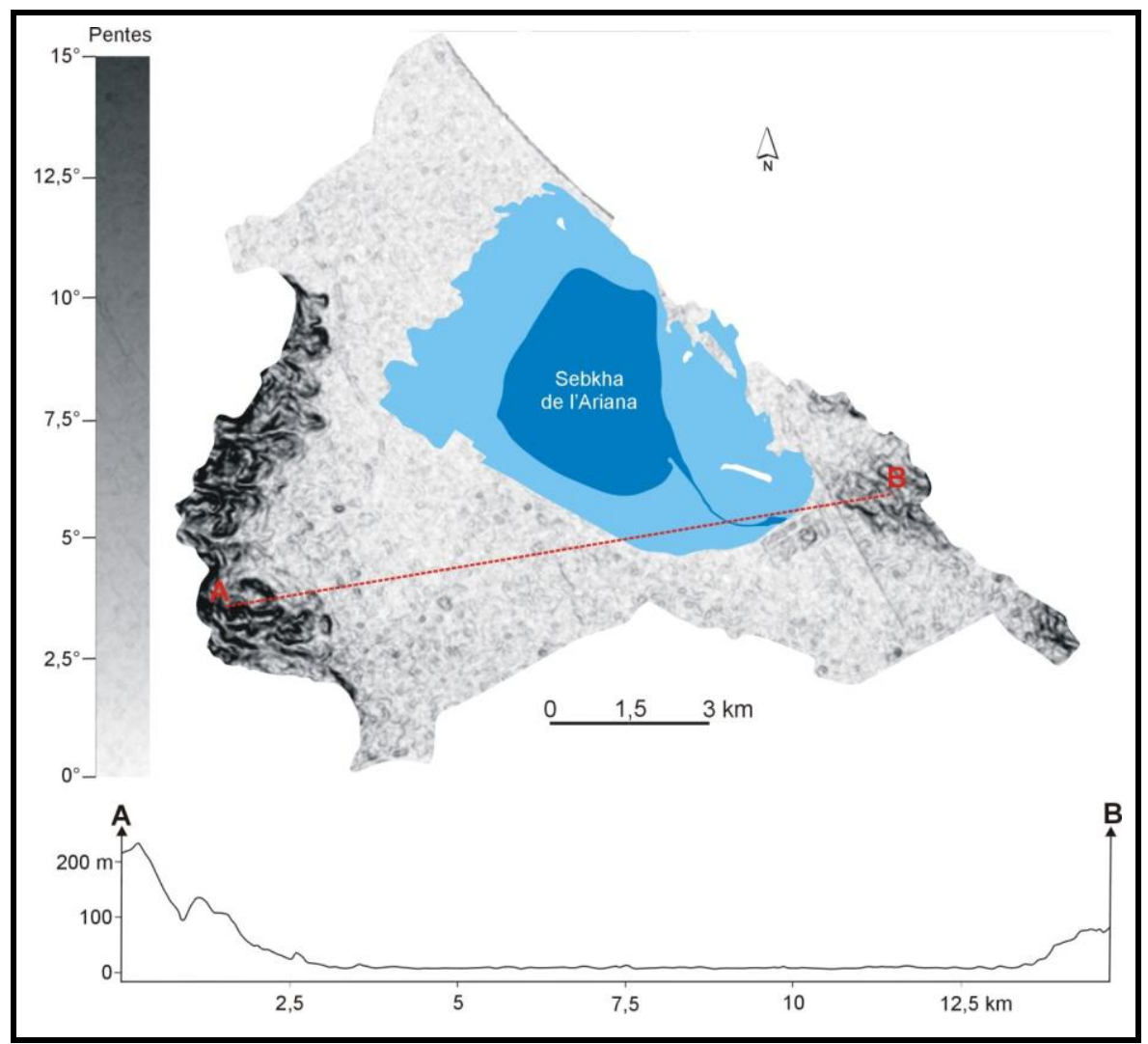

Fig. (2): Les classes des pentes dans le bassin versant de la sebkha de l'Ariana.

Sources: Une image ASTER (données d'altitude à couverture globale, résolution spatiale $=20 \mathrm{~m}$ ) et des cartes topographiques type 1982 au 1/25000 (feuille ${ }^{\circ}$ XIII «NE et SE» et feuille ${ }^{\circ} \mathrm{XIV} \ll \mathrm{NO}$ et $\left.\mathrm{SO} »\right)$. 
Ainsi, en amont, bien qu'il s'agisse d'un ensemble collinaire peu élevé, les pentes sont courtes mais raides et découpées par de nombreuses vallées étroites et souvent profondes dépassant parfois la quarantaine de mètres et taillées dans un matériel marno-argileux tendre. En aval, les oueds arrivent souvent à inonder et alluvionner la plaine des rives est de la sebkha.

Le fond du bassin versant est constitué par une sebkha d'une forme ovale et occupe une superficie d'environ $50 \mathrm{Km}^{2}$. Le fond de la sebkha se situe à quelques centimètres au-dessous du niveau marin. La sebkha est délimitée du côté est et sud-est, respectivement par un cordon littoral qui s'étend de l'embouchure de la Majerda au Nord au cap Gammart au Sud et par les collines de Sidi Bou Saïd (129 m) et de Gammart (104 m). Plusieurs écoulements descendent des collines littorales allant de Carthage à Gammart, principalement l'oued Sidi Bou Saïd et l'oued Sidi Daoued au Sud-Est, l'oued Tabeuk et les oueds Sidi Rahal et El Khaoui au Nord (Fig. 1).

Du côté ouest, le bassin versant est délimité par un alignement à orientation méridienne et à pentes fortes, des unités morphologiques des Rous El Hraïeg, Jbel Nahli, Jbel Ayari et Jbel Sidi Amor à 1'Ouest. Cet alignement et bien que de faible altitude ne dépassant pas $236 \mathrm{~m}$, il est sillonné par une multitude d'oueds intermittents parfois profonds dont les plus importants sont les oueds Ennkhilet et Borj Torki qui atteignent le rang 5 selon la hiérarchisation de Stahler 1957.

À l'Ouest, la plaine de l'Ariana est très basse et elle est coincée entre les versants et la sebkha. À l'Ouest et au Nord et au Sud, la topographie est généralement plane et monotone. La plaine de Soukra-Choutrana-Ariana au Sud se caractérise par des altitudes très basses et une surface presque plane puisque l'altitude maximale ne dépasse pas $7 \mathrm{~m}$. Cette surface est parsemée par des cuvettes mal drainées formant des zones inondables. Les quelques cours d'eau qui drainent cette plaine, de plus en plus peuplée, jouent le rôle d'un évacuateur des eaux pluviales vers la sebkha.

L'absence d'une zone intermédiaire entre les sommets des Jbels environnants et la plaine engendre généralement un écoulement violent. Les oueds peuvent être très actifs à l'occasion des phénomènes pluviométriques importants.

La carte géologique au $1 / 50000^{\text {ème }}$ montre que la structure du Jbel Nahli est constituée par des formations qui s'échelonnent du Crétacé inférieur au Quaternaire. La colonne lithostratigraphique synthétique de la zone reflète bien une prédominance en faveur des formations meubles de type marnes. Les formations quaternaires sont très bien développées sur les versants et dans la plaine. Un affleurement de conglomérats subhorizontaux alterne avec des argiles sableuses rougeâtres. Les éléments constituant les conglomérats 
sont variés et proviennent de la série Crétacé supérieur-Éocène affleurant à proximité. Les alluvions récentes recouvrent la plaine. Elles sont représentées par des argiles et par des sables argileux à concrétions de gypse et de calcaire. Du point de vue de la pédologie, la plaine de Choutrana est constituée par des terres lourdes argileuses ou limono-argileuses. En s'approchant de la sebkha, les marques d'hydromorphie, provoquées par la stagnation des eaux de ruissellement et la remontée du toit de la nappe phréatique, sont nombreuses.

La carte hydrogéologique de la zone montre que sur les rives ouest et sud-ouest de la sebkha, la nappe phréatique qui communique directement avec la sebkha, se présente en trois secteurs. Un premier secteur où la nappe est affleurante et qui correspond aux rives de la sebkha et aux marécages de la station d'épuration de Choutrana. Un deuxième secteur où la nappe est à moins de $5 \mathrm{~m}$ de profondeur et qui correspond à la plaine de Choutrana. Un troisième secteur où la nappe est à plus de $5 \mathrm{~m}$ de profondeur et qui correspond au piedmont du Jbel Nahli. Du côté de Soukra, sur les rives sudouest de la sebkha, la nappe phréatique est aussi affleurante et les terres sont constamment gorgées d'eau.

Pour caractériser la climatologie de la région, nous avons examiné une série pluviométrique de 50 ans couvrant la période de 1953 à 2003. Nous avons pris comme référence la moyenne pluviométrique annuelle de 445,96 mm de la station la plus proche, celle d'Ariana (station $\left.n^{\circ} 43927\right)$. La série examinée donne un diagramme simple reflétant une variation saisonnière importante dans la répartition annuelle des précipitations avec une nette concentration entre le mois de septembre et le mois d'avril et avec un pic au niveau du mois de janvier de l'ordre de $65,32 \mathrm{~mm}$.

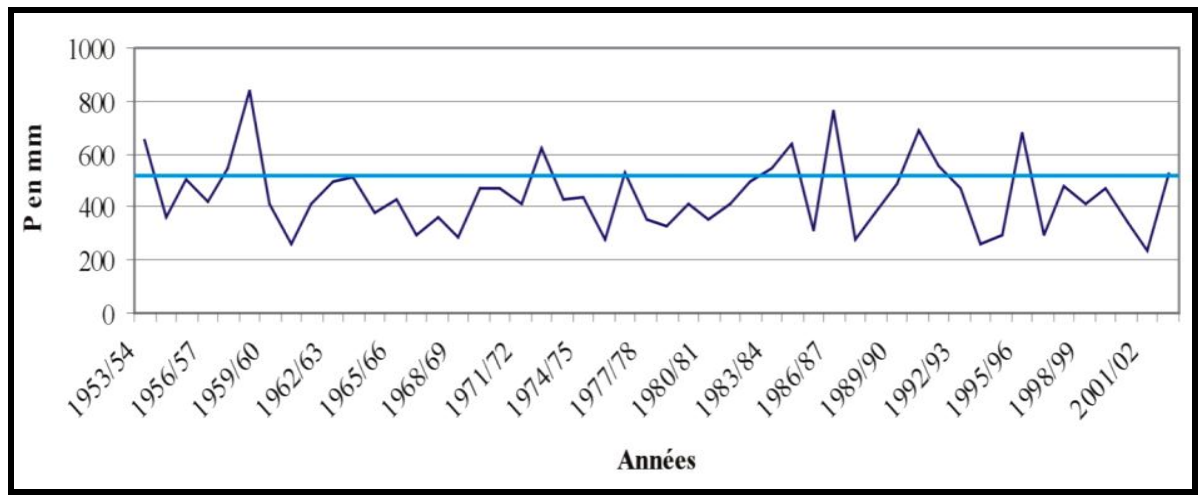

Fig. (3): Variation de la pluviométrie annuelle moyenne à la station d'Ariana entre 1953 et 2003.

Source: données brutes de l'Institut National de Météorologie 
L'automne qui cumul $36 \%$ de la pluie annuelle se caractérise par des précipitations souvent sous forme d'orages de courte durée mais, à fortes intensités qui engendre un écoulement torrentiel vers la plaine (Chouari, 2006 et 2009).

Ainsi, les alimentations naturelles de la sebkha sont assurées par les précipitations, les cours d'eaux (oued Ennkhilet, oued Sidi Amor, oued Tabeuk, oued El Khaoui...), les eaux marines et les eaux souterraines. La sebkha est aussi l'unique exutoire, où se décharge la nappe souterraine. La communication entre la mer et la nappe est très limitée (faible gradient et faible perméabilité; SWECO-APAL, 2000). Les exportations sont assurées par l'infiltration, l'évaporation et le sous-écoulement vers la mer. Récemment une communication avec la mer a été assurée par des canaux et une station de pompage placée au niveau d'une interruption du cordon littoral. La communication naturelle de la sebkha avec la mer n'est qu'occasionnelle pendant les tempêtes, puisque le canal est souvent obturé par les sables accumulés par le vent et les vagues.

Les calculs de l'équilibre hydrique montrent que la sebkha reçoit aujourd'hui environ $15 \%$ d'eau de la nappe, $40 \%$ des eaux de ruissellement (dont la moitié provient des débordements des eaux usées) et $45 \%$ de l'eau de pluie tombant directement dans la sebkha (SWECO-APAL, 2000).

\section{Des modes d'urbanisation aggravant la vulnérabilité et des aménagements hydrauliques incapables de faire transiter les quantités d'eau déferlantes}

L'explosion démographique d'après l'indépendance du pays a poussé la zone d'étude vers une extension importante du tissu urbain. Le tableau cidessous (Tab. 1), donne une idée sur l'évolution de la population dans les principales délégations du bassin versant.

Tab. (1): Évolution de la population dans les délégations du bassin versant de la sebkha de l'Ariana.

\begin{tabular}{|l|c|c|c|}
\hline Municipalité & Ariana & Soukra & Raoued \\
\hline Population 1994 & 70694 & 54155 & 33140 \\
Densité 1994 $\left(\mathrm{hab} / \mathrm{km}^{2}\right)$ & 5611 & 2881 & 524 \\
\hline Population 2004 & 97687 & 89151 & 60896 \\
Densité 2004 $\left(\mathrm{hab} / \mathrm{km}^{2}\right)$ & 7753 & 4742 & 964 \\
\hline Population 2010 & 109211 & 107779 & 77580 \\
Densité 2010 $\left(\mathrm{hab} / \mathrm{km}^{2}\right)$ & 8917 & 5727 & 1905 \\
\hline Population 2014 & 119693 & 129693 & 94961 \\
Densité 2014 $\left(\mathrm{hab} / \mathrm{km}^{2}\right)$ & 9772 & 6891 & 2331 \\
\hline
\end{tabular}

Source: Institut National de la Statistique, 2014. 
Il s'agit de délégations d'Ariana, Soukra et Raoued qui ont connu respectivement des taux de croissance entre 1994 et 2014 de l'ordre de $69,3 \%, 139,5 \%$ et $186,5 \%$, et des taux annuels moyens de l'ordre de $0,81 \%$, $1.67 \%$ et $3,16 \%$.

L'extension actuelle de la tâche urbaine se fait d'ores et déjà au détriment des terres basses gorgées d'eau. La ville a tendance à s'étendre en " grignotant » les plaines agricoles environnantes et les terres à dominance de culture maraîchère et annuelle se trouvent chaque hiver gorgées d'eau et leur exploitation est devenue très difficile sans drainage. Le suivi de l'occupation des sols permet de comprendre les étapes de cette extension aussi bien à l'échelle spatiale que temporelle. La figure $\mathrm{n}^{\circ} 4$ (2016), effectuée sur un fond au $1 / 2000^{\mathrm{eme}}$ afin de mieux cerner l'occupation du sol, montre l'éloignement des banlieues où les terrains sont à bas prix. Les constructions arrivent au bout d'un alignement des Jbels qui constituaient le cadre naturel du bassin versant. Quant aux collines littorales allant de Carthage à Gammart, elles connaissent une extension urbaine rapide qui se fait vers l'Ouest dans les zones basses limitrophes à la route RVE 550 et aux dépens des terres agricoles de la plaine de Bhar Lazreg. La rapide extension urbaine a été aussi favorisée par l'élargissement des voies principales vers Bizerte, Raoued et la Soukra et l'installation conséquente des activités de services induisant par effet d'entrainement une densification accrue du tissu urbain (Fig. 4).

Suite à cette extension urbaine, les sections d'oueds sont souvent remplacées en aval sur les rives de la sebkha par des canaux de drainage à profil rectangulaire ou trapézoïdal et à parois en béton (Oueslati, 1999, Zarai, 2000, 2006; Dlala, 2007; Chouari, 2003, 2009 et 2015; Fehri et Mathlouthi, 2012). Dans beaucoup de cas, les ouvrages en question sont de nos jours sous-dimensionnés par rapport aux volumes d'eau susceptibles de s'écouler et les sections des oueds se trouvent envahies par les constructions qui jouent, souvent, le rôle d'obstacles qui empêchent l'écoulement naturel des cours d'eau vers la plaine et la sebkha. À titre d'exemple, dans la plaine, l'oued Ennkhilet se raccorde à deux canaux de drainage. Le premier draine la partie sud de la cité El Ghazala et le deuxième prend naissance au niveau des hauteurs situées à la limite Nord-Ouest du sous-bassin versant du même nom. Ces tronçons d'amont sont aménagés sous forme de dalots et ceux à l'aval sont des canaux à ciel ouvert avec une largeur au fond d'environ $10 \mathrm{~m}$. L'oued intercepte aussi au niveau de sa partie aval un canal de drainage en terre. Il est barré dans la partie amont par la retenue collinaire d'Aïn Snoussi (plantée au parc Ennahli). 


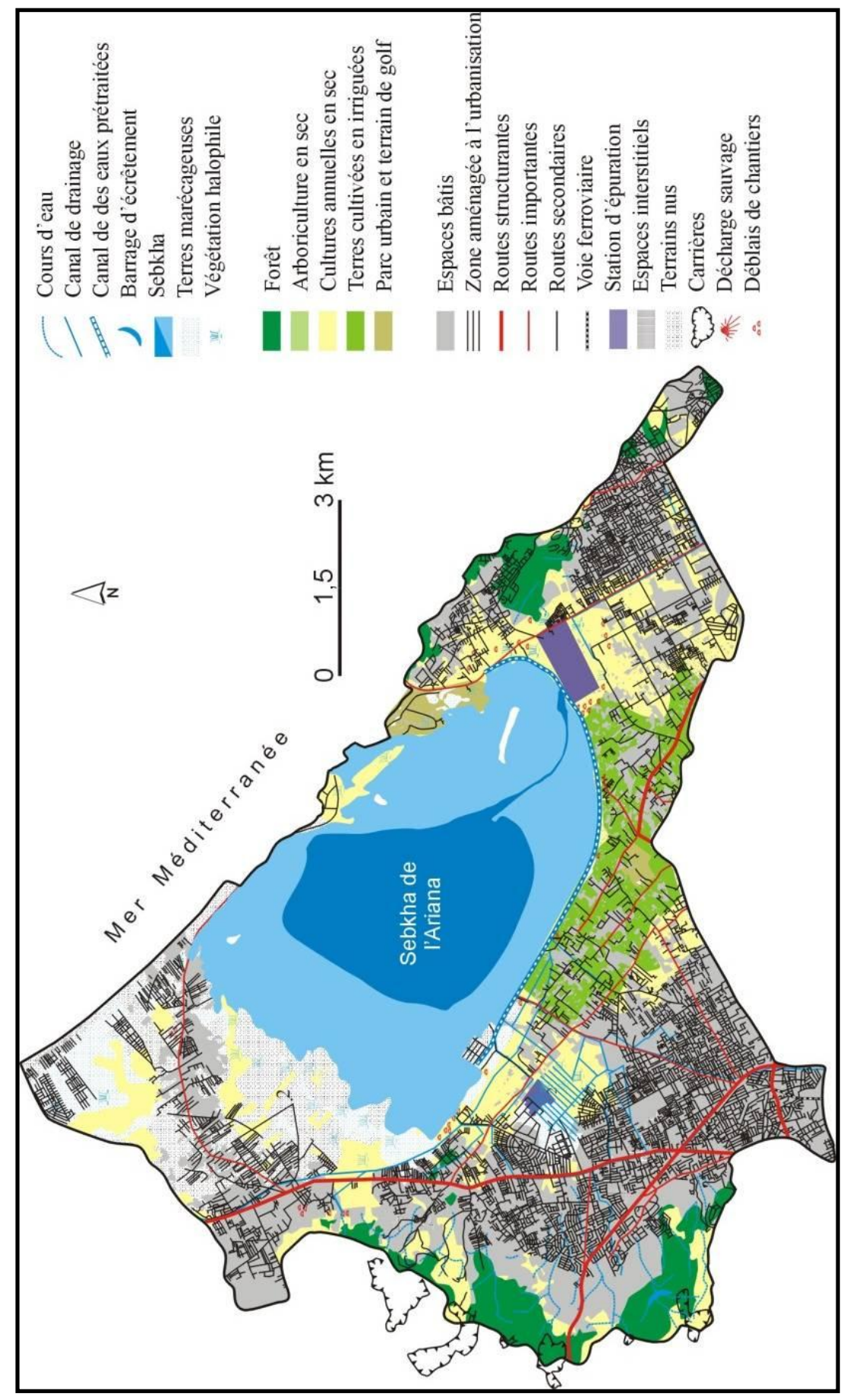

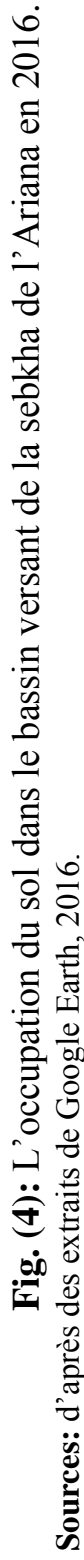


Les inondations des années précédentes témoignent de la faillite et de l'incapacité du réseau existant à contenir la lame d'eau ruisselée. Actuellement, suite à l'imperméabilisation des terrains de dernières années, à pluviométrie égale aux épisodes précédents, les réseaux d'évacuation des eaux pluviales seront amenés à drainer des quantités d'eau toujours plus importantes. Les inondations des dernières années $(2003,2007$ et 2011) nous apprennent, qu'en dépit de leur taille, nous avons désormais affaire à des cours d'eau de moins en moins maitrisables et pour cause, des interventions anthropiques qui, dans la plupart des cas ne tiennent pas compte du caractère imprévisible de ces oueds si petits soient-ils (Oueslati, 1999, Chouari, 2006 et 2009 et Fehri et Mathlouthi, 2012).

L'enchaînement d'un ensemble de mécanismes générateurs des inondations et surtout leur rapidité et leur localisation amènent à distinguer plusieurs types de submersions d'eau dans le bassin versant de la sebkha de l'Ariana.

\section{Un cumul d'imprudence dans le bassin versant de la sebkha de l'Ariana}

Lors des pluies importantes à exceptionnelles (2003, 2007, 2011), les torrents (oued Ennkhilet, oued Tabeuk,...) à pente forte (supérieure à $6 \%$ ) descendent des versants environnants et provoquent des inondations. Ceci témoigne de l'importance des coups de chasse d'eau qui peuvent s'y produire et de la soudaineté de l'écoulement. Les eaux concentrées depuis les versants se trouvent, dans la plupart des cas, obligées d'emprunter les rues et de se rassembler derrière les obstacles provoquant des stagnations d'impressionnantes quantités d'eaux et de grands volumes de sédiments déposés sur les trottoirs et les chaussées des quartiers. La figure $\mathrm{n}^{\circ} 5$ cidessous, présente de façon schématique, les mouvements horizontaux des eaux, à partir du Jbel Nahli vers la mer méditerranée.

En traversant des zones basses et déprimées, les petits oueds trouvent les conditions topographiques favorables pour déborder et envahir ses environs provoquant l'inondation de larges espaces (Photos 1 et 2). Dans les plaines, parfois nous avons l'impression que l'absence d'un écoulement permanent dans le réseau naturel de drainage superficiel a conduit à oublier son rôle en période d'orage, voire sa présence. Le drainage urbain de la plaine d'Ariana, à titre d'exemple, consiste en des réseaux de drainage pluvial et des réseaux d'eaux usées. Ce drainage, essentiellement réalisé et contrôlé par le concours de l'ONAS (Office National d'Assainissement) et le Ministère de l'Équipement et de l'Habitat (Direction de l'Hydraulique Urbaine), paraît insuffisant parce que les inondations des routes urbaines sont fréquentes même pour des pluies ordinaires. Lors d'orages très intenses pour lesquels le réseau souterrain est insuffisant, les ruissellements se font en surface et la voirie se transforme en cours d'eau. 


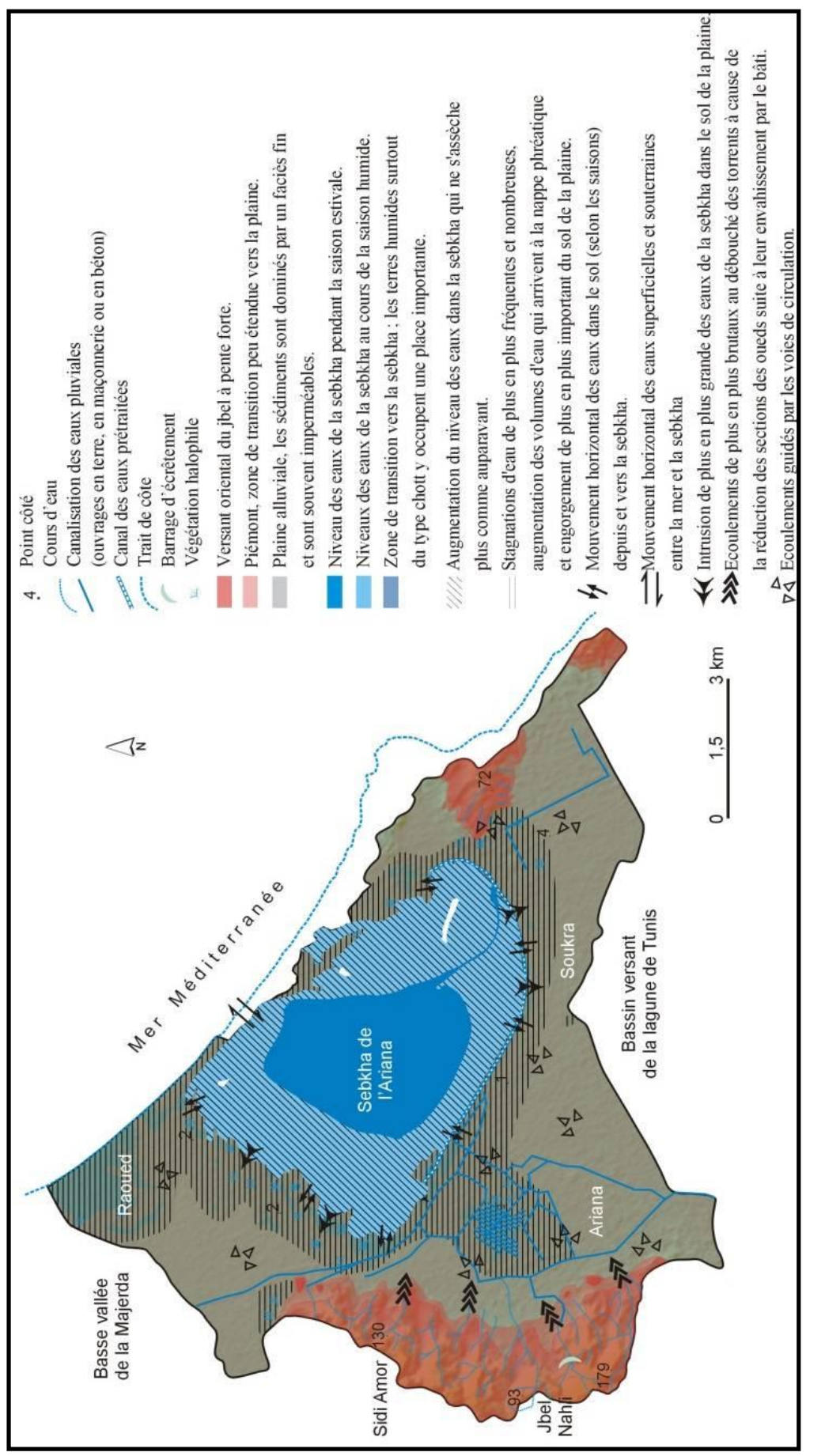

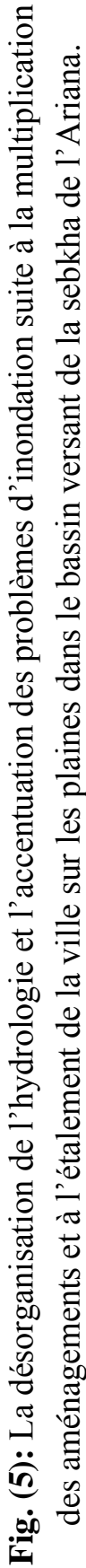




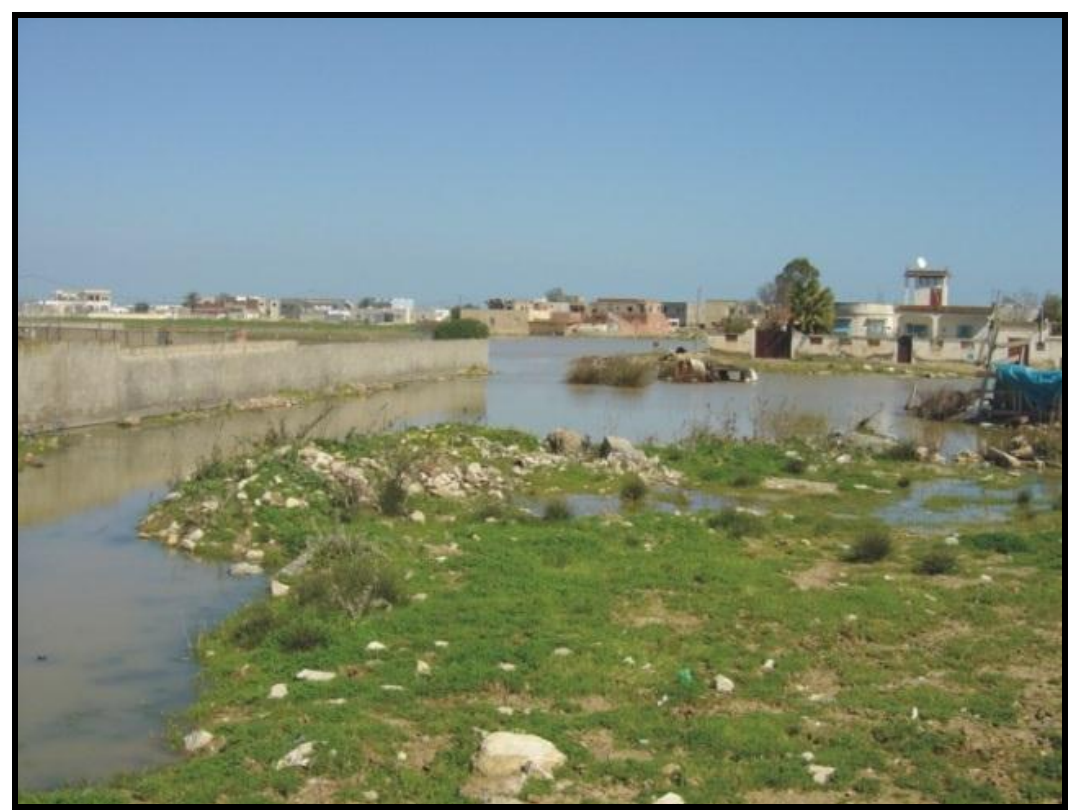

Photo 1. Raoued: les terres basses jadis réservées à l'agriculture sont aujourd'hui urbanisées et désormais à risque d'inondation (W. Chouari, février 2006).

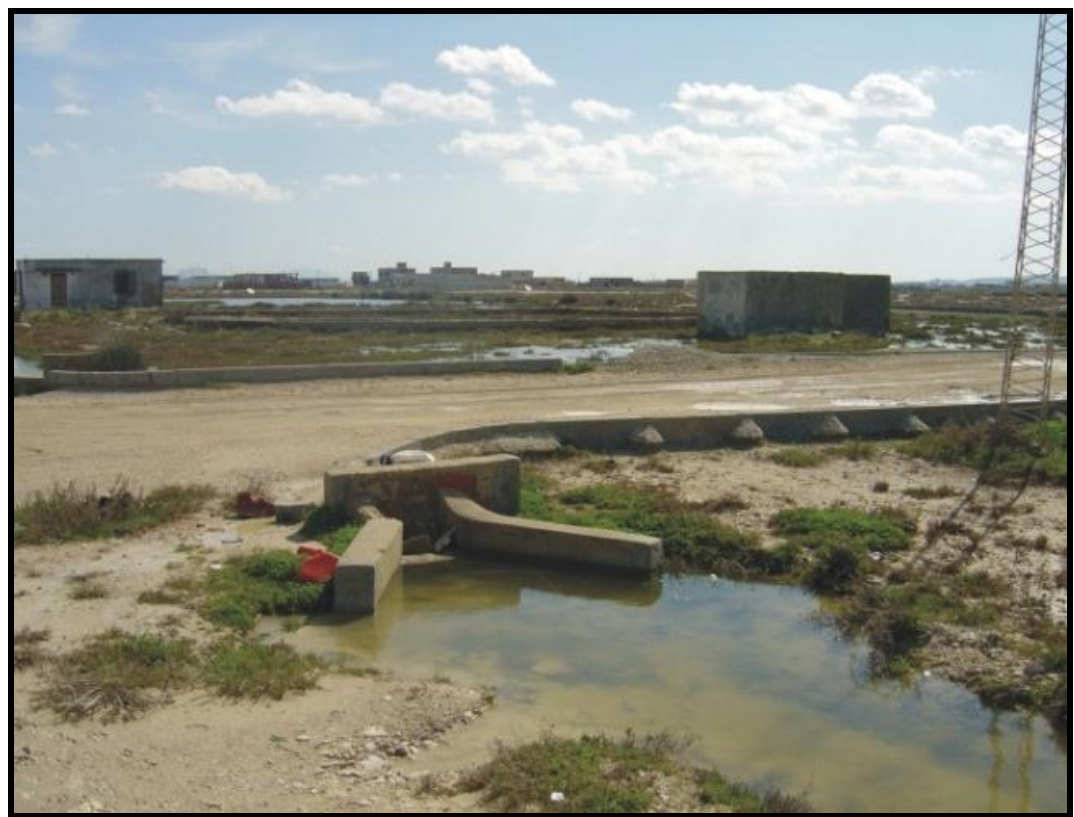

Photo 2. Des lotissements sur un terrain de type sebkha et chott gorgé d'eau à Raoued-plage (W. Chouari, février 2006). 
Par ailleurs, les lits des écoulements qui descendent des collines littorales se perdent à l'aval où l'urbanisation fait obstacle à l'évacuation naturelle des eaux vers la sebkha de l'Ariana. Les collecteurs et canaux EP existants, notamment celui de la zone du stade, des cités Tabeuk, de la rue Hédi Chakeur, des cités Rmila et le canal Tabeuk, principal émissaire de rejet, sont de faible capacité et généralement comblés de dépôts solides (Chouari, 2009).

Dans un espace si occupé, les effets du mode d'occupation du sol ne sont pas moins importants que les effets induits par l'irrégularité topographique dans la canalisation des écoulements. Même s'il est difficile de représenter les chemins suivis par les eaux débordantes à travers les blocs d'habitations autrement que de façon schématique, il paraît évident que des axes d'écoulement préférentiels se distinguent sur le terrain surtout lorsque les canalisations se révèlent bien souvent incapables d'acheminer les apports d'eaux supplémentaires induits par les ruissellements sur les nouvelles surfaces imperméabilisées raccordées aux réseaux.

Par ailleurs, jusqu'à une période récente, le comportement des nappes et le fonctionnement hydrologique de l'ensemble de l'écosystème suivait un cycle régulier, caractérisé par un assèchement complet de la sebkha durant l'été, favorisant ainsi le drainage des nappes. En hiver, les nappes se rechargeaient à travers les formations perméables des versants environnants et les sols de texture grossière de la plaine de Soukra (dune de Soukra). Cet équilibre n'était que rarement perturbé, même durant les années de pluies exceptionnelles. Au début des années 1980 démarrent les problèmes de stagnation des eaux, de remontée de la nappe et d'engorgement des terres (Oueslati, 1999 et Chouari, 2009).

La sebkha reçoit aussi de grandes quantités d'eau depuis les stations d'épuration (Choutrana et la Côtière Nord) lorsqu'elles sont saturées, auxquelles s'ajoute un apport considérable assuré par les fosses perdues dont les eaux arrivent jusqu'à la nappe. À partir de 1985, les affleurements d'eau souterraine sont devenus plus fréquents dans les zones basses (altitude $=5 \mathrm{~m}$ et pente $<2 \%$ ) et ont favorisé l'extension des zones inondées. Ainsi, pendant les périodes pluvieuses et dans plusieurs endroits des rives, notamment à Soukra et à Raoued, il est difficile de distinguer entre le niveau supérieur de la nappe et le niveau bas des eaux de surfaces.

En outre, dans le bassin versant de la sebkha de l'Ariana, les submersions marines lors des tempêtes affectent des terrains situés en dessous du niveau de plus hautes mer. La potentielle élévation du niveau de la mer au cours du siècle à venir aura de profondes répercussions sur les systèmes naturels et aménagés et sur la qualité de la vie dans la zone. Les impacts pourraient être importants en absence de mesures préventives. À 
cette submersion, il faut aussi ajouter l'effet de l'érosion marine qui se fera entre temps ainsi que l'effet d'une subsidence de plus en plus confirmée par les géologues (Chouari, 2009 and Ennesser et al., 2011).

Les évènements pluviométriques de dernières années (2003, 2007 et 2011) ont montré que les infrastructures de drainage et de protection contre les inondations existantes n'étaient pas totalement efficaces. Dans tous les cas, les infrastructures de lutte contre les inondations existantes ou projetées ne peuvent pas assurer une protection totale des zones inondables. Seule une politique urbaine concertée et globale le permettra. Le fait de diriger l'extension urbaine vers des zones non inondables repérées par des cartes thématiques par l'outil SIG (Système d'Information Géographique), peut prévenir les problèmes et éviter les dégâts.

\section{II) La cartographie d'inondations dans le bassin versant de la sebkha Ariana}

Afin de délimiter les zones inondables et de définir les caractéristiques de l'inondation, nous avons créé un Système d'Information Géographique (SIG) de la zone et nous avons réalisé des calculs hydrologiques $(\mathbf{K r})$.

\section{Estimation des coefficients de ruissellement}

Le bassin versant de la sebkha de l'Ariana est de plus en aménagé et peuplé. Le tableau suivant montre que le taux d'imperméabilisation du bassin versant est estimé en l'état actuel entre $38 \%$ et $52 \%$ (Tab. 2).

Tab. (2): Les coefficients d'imperméabilisation dans les sous-bassins versant de la sebkha de l'Ariana.

\begin{tabular}{|l|c|c|}
\hline \multicolumn{1}{|c|}{ Bassin versant } & Surface en H & $\begin{array}{c}\text { Taux d'imperméabilisation } \\
\text { en situation actuelle }\end{array}$ \\
\hline Ariana & 3087 & $38 \%$ \\
\hline Bhar lazreg-Marsa & 2233 & $24 \%$ \\
\hline Soukra & 940 & $38 \%$ \\
\hline Raoued & 2483 & $52 \%$ \\
\hline
\end{tabular}

Source: Ennesser et al., 2011.

Pour estimer les coefficients de ruissellement dans le bassin versant nous nous sommes appuyés sur le modèle $« S C S^{(1)}{ }$. Le coefficient de ruissellement est le rapport entre la hauteur d'eau ruisselée à la sortie d'une surface considérée, dite «pluie nette $(\mathrm{R})$ » et la hauteur d'eau précipitée, dite «pluie brute $(\mathrm{P}) »$.

(1) "Soil Conservation Service" développé par le service de conservation des sols de l'USA. 


\section{$\mathrm{Kr}=\mathbf{R} / \mathbf{P} \times 100$}

Ce modèle relie la pluie nette $(\mathrm{R})$ à la pluie brute $(\mathrm{P})$, par l'équation suivante (Mockus, 1972; Baltas et al., 2007):

Où,

$$
\mathbf{R}=(\mathbf{P}-\mathbf{I a})^{2} /[(\mathbf{P}-\mathbf{I a})+\mathrm{S}]=(\mathbf{P}-\mathbf{0}, 2 \mathrm{~S})^{2} /(\mathbf{P}+\mathbf{0 , 8 S})
$$

$\mathrm{R}$ = la pluie ruisselée $(\mathrm{mm})$;

$\mathrm{P}=$ les précipitations $(\mathrm{mm})$.

Les paramètres d'ajustement du modèle sont les suivants.

$I a=$ les pertes en début d'évènement (par interception), elles sont égales à $0,2 \mathrm{~S}$.

$\mathrm{S}$ = la capacité maximale de rétention en eau du réservoir sol. Elle est exprimée en $(\mathrm{mm})$ et peut être estimée en appliquant la formule suivante:

$$
\mathrm{S}=(25400 / \mathrm{CN})-254
$$

Où, le Curve Number du modèle SCS est déterminé à partir d'un tableau (Tab. 3) pour plusieurs types de sols et de couvertures végétales. Le CN est inversement proportionnel à S. Plus la valeur du CN tend vers 100, plus le sol est imperméable (Mishra \& Singh, 2003 et Fehri \& Zahar, 2016).

Le calcul du coefficient de ruissellement est réalisé via une application élaborée à l'aide du module Spatial Modeler d'ERDAS_IMAGINE. Pour caractériser des coefficients de ruissellement, on procèdera au croisement de deux paramètres à savoir, les pluies, et le rendement hydrologique de chaque type d'occupation du sol caractérisé par les Curve Number $(\mathrm{CN})$ du modèle SCS (Soil Conservation Service of USA); (Mishra et al., 2003). Le résultat de ce croisement est une répartition spatiale des coefficients de ruissellement basée sur des classes variant de faible à très fort à l'échelle de tout le bassin versant. Pour chaque type d'occupation du sol nous avons défini un coefficient de ruissellement. Et, le choix du «Curve Number» relatif à chaque type d'occupation est pris en se basant sur les résultats de calculs planimétriques des Coefficients d'Occupation du Sol «COS» effectués sur des zones tests.

Le coefficient de ruissellement est une variable aléatoire liée à l'imperméabilité et à la hauteur de la pluie. Sur les versants, la répartition surfacique des groupes des sols dans le bassin versant montre la prédominance des sols à potentiel de ruissellement fort, c'est-à-dire des sols minéraux bruts des versants (lithosols et régosols). Des sols halomorphes caractérisent les rives Est, Nord et Nord-Ouest de la sebkha et des sols peu évolués d'apport s'étendent sur ses rives Sud-Ouest. Nous avons attribué 
pour chaque type d'occupation des sols un coefficient de ruissellement qui lui correspond (Fig. 6).

Tab. (3): Composés hydrologiques de la couverture du sol et numéros associés pour des conditions moyennes d'une aire de drainage (Mishra et Singh, 2003).

\begin{tabular}{|c|c|c|c|c|c|c|}
\hline \multirow{2}{*}{$\begin{array}{l}\text { Usage du } \\
\text { terrain ou } \\
\text { couverture }\end{array}$} & \multirow{2}{*}{$\begin{array}{c}\text { Traitement du sol ou } \\
\text { pratique }\end{array}$} & \multirow{2}{*}{$\begin{array}{c}\text { État } \\
\text { hydrologique }\end{array}$} & \multicolumn{4}{|c|}{$\begin{array}{l}\text { Sol du groupe } \\
\text { hydrologique }\end{array}$} \\
\hline & & & A & $\mathrm{B}$ & $\mathrm{C}$ & $\mathrm{D}$ \\
\hline Jachère & Lignes droites & Mauvais & 77 & 86 & 91 & 94 \\
\hline \multirow{5}{*}{$\begin{array}{l}\text { Cultures en } \\
\text { lignes }\end{array}$} & Lignes droites & Mauvais & 72 & 81 & 88 & 91 \\
\hline & Lignes droites & Bon & 67 & 78 & 85 & 89 \\
\hline & En courbes de niveau & Mauvais & 70 & 79 & 84 & 88 \\
\hline & En courbes de niveau & Bon & 65 & 75 & 82 & 86 \\
\hline & $\begin{array}{l}\text { En courbes de niveau } \\
\text { et terrasses }\end{array}$ & Mauvais & 66 & 74 & 80 & 82 \\
\hline \multirow[t]{6}{*}{ Céréales } & Lignes droites & Mauvais & 65 & 76 & 84 & 88 \\
\hline & Lignes droites & Bon & 63 & 75 & 83 & 87 \\
\hline & En courbes de niveau & Mauvais & 63 & 74 & 82 & 85 \\
\hline & En courbes de niveau & Bon & 61 & 73 & 81 & 84 \\
\hline & $\begin{array}{l}\text { En courbes de niveau } \\
\text { et terrasses }\end{array}$ & Mauvais & 61 & 72 & 79 & 82 \\
\hline & $\begin{array}{l}\text { En courbes de niveau } \\
\text { et terrasses }\end{array}$ & Bon & 59 & 70 & 78 & 81 \\
\hline \multirow{6}{*}{$\begin{array}{l}\text { Cultures denses } \\
\text { de légumineuses } \\
\text { ou prairie en } \\
\text { rotation }\end{array}$} & Lignes droites & Mauvais & 66 & 77 & 85 & 89 \\
\hline & Lignes droites & Bon & 58 & 72 & 81 & 83 \\
\hline & En courbes de niveau & Mauvais & 64 & 75 & 83 & 85 \\
\hline & En courbes de niveau & Bon & 55 & 69 & 78 & 83 \\
\hline & $\begin{array}{l}\text { En courbes de niveau } \\
\text { et terrasses }\end{array}$ & Mauvais & 63 & 73 & 80 & 83 \\
\hline & $\begin{array}{l}\text { En courbes de niveau } \\
\text { et terrasses }\end{array}$ & Bon & 51 & 67 & 76 & 80 \\
\hline \multirow{6}{*}{$\begin{array}{l}\text { pâturage naturel } \\
\text { ou parcours }\end{array}$} & Naturel & Mauvais & 69 & 79 & 86 & 89 \\
\hline & Naturel & Moyen & 49 & 69 & 79 & 84 \\
\hline & Naturel & Bon & 39 & 61 & 70 & 80 \\
\hline & En terrasses & Mauvais & 47 & 67 & 81 & 88 \\
\hline & En terrasses & Moyen & 25 & 59 & 75 & 83 \\
\hline & En terrasses & Bon & 6 & 35 & 70 & 79 \\
\hline \multicolumn{2}{|c|}{ Prairie (permanent) } & Bon & 30 & 58 & 71 & 78 \\
\hline \multirow{3}{*}{\multicolumn{2}{|c|}{ Forêt }} & Mauvais & 45 & 66 & 77 & 83 \\
\hline & & Moyen & 36 & 60 & 73 & 79 \\
\hline & & Bon & 25 & 55 & 70 & 77 \\
\hline \multicolumn{2}{|l|}{ Bâtiments } & ------ & 59 & 74 & 82 & 86 \\
\hline \multicolumn{2}{|c|}{ Pistes poussiéreuses } & ------ & 72 & 82 & 87 & 89 \\
\hline \multicolumn{2}{|c|}{ Surface dure } & ----- & 74 & 84 & 90 & 92 \\
\hline \multicolumn{2}{|c|}{ Surfaces imperméables } & ------ & 100 & 100 & 100 & 100 \\
\hline \multicolumn{2}{|c|}{ Surfaces d'eau (étang, lac, ...) } & ----- & 100 & 100 & 100 & 100 \\
\hline
\end{tabular}


Le bassin versant se caractérise par des pentes fortes et des vallées étroites et profondes sur les versants et par une extension importante des terrains imperméables dans la plaine. Ces caractéristiques conjuguées avec le régime d'écoulement des oueds contribuent au renforcement du coefficient de ruissellement et des quantités d'eau évacuées vers l'exutoire constitué par la sebkha de l'Ariana. La répartition spatiale des coefficients de ruissellement sur les versants montre la prédominance des surfaces présentant des coefficients de ruissellent faibles à moyens qui sont compris entre $17 \%$ et $55 \%$. Les coefficients très forts et qui dépassent $70 \%$ sont observés au niveau des voies routières (voies principales et secondaires) et au centre de la ville de l'Ariana complètement imperméabilisé par le bitume et le béton.

Pour caractériser l'aléa nous nous sommes contentés des observations des limites des champs inondés, de la durée de la submersion et des quelques indicateurs des hauteurs atteintes par les eaux, faites sur le terrain ou par photo-interprétation au cours de trois évènements principaux $(2003,2007$ et 2011).

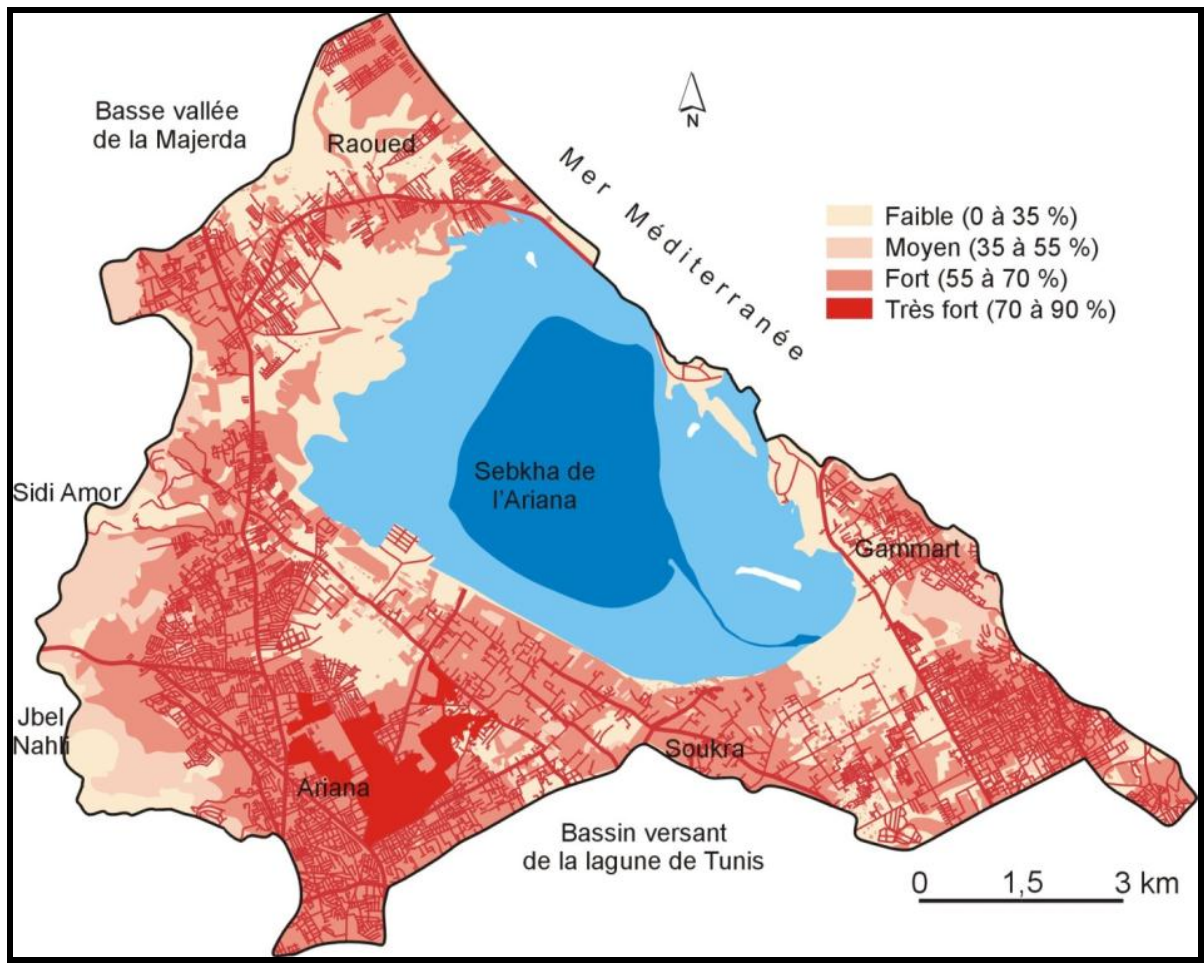

Fig. (6): Les coefficients de ruissellement pour chaque type d'occupation selon les groupes hydrologiques du sol. 


\section{La cartographie des aléas}

La carte d'aléa est un document de synthèse qui présente la valeur des différents paramètres caractéristiques des inondations retenus: durée de la submersion et types d'inondations. Faute de l'absence d'un cours d'eau structurant le bassin versant et crainte de tomber dans l'empirique, nous nous sommes contentés des observations des limites des champs inondés et des quelques indicateurs des hauteurs atteintes par les eaux, faites par moi-même ou par mes prédécesseurs par photo-interprétation au cours de trois évènements principaux $(2003,2007$ et 2011) ou par le recueil de témoignages auprès des habitants. Nous avons aussi essayé d'intégrer des données qui font partie de l'hydrologie et de l'hydraulique, complétés par une analyse par photo-interprétation et des observations directes sur le terrain. Certains indices forment souvent d'excellentes limites à l'eau. Les routes surélevées offrent une barrière topographique artificielle importante pour limiter l'extension horizontale des eaux de débordement. D'autres représentent aussi des marqueurs à prendre en considération mais avec un faible taux de précision et de fiabilité à l'image de certaines irrégularités topographiques. Ainsi, dans le bassin versant de la sebkha de l'Ariana une inondation rapide peut être renforcée par un ruissellement urbain. De plus, l'aléa inondation prend un aspect particulier du fait de la topographie, de la morphologie urbaine et d'autres facteurs à degré d'aggravation du phénomène plus au moins important.

Même si nous travaillons à l'échelle des sous-bassins versants, la localisation avec précision de la ligne naturelle des hautes eaux par photointerprétation est une tâche difficilement réalisable correctement. La situation devient beaucoup plus difficile dans l'espace urbain ou dans des espaces fortement exploités par l'agriculture, même à une grande échelle (1/5000). Par ailleurs, la superposition des crues des petits oueds des versants avec les écoulements issues des routes goudronnées nécessite le recours à une étude hydraulique plus fine qui prend en considération dans une approche systémique le sous-bassin versant local et la plaine alluviale aménagée. Ces difficultés nous ont poussées à adopter une méthode cartographique basée sur le carroyage qui consiste à représenter les résultats d'interprétations des photographies aériennes, des observations du terrain et des entretiens auprès de la population..., sur la base d'un carroyage normalisé. Cette normalisation permet d'offrir des potentialités de traitements qui prennent en compte la donnée spatiale, liée à l'occupation des sols et à l'inondation, de façon homogène. La trame du carroyage, composée de carreaux de $100 \mathrm{~m}$ de côté, est calée sur un assemblage des extraits tirés de Google Earth 2016 et sur la mosaïque reconstituée des photographies aériennes au 1/5000 de l'année 2002. À chaque maille est affectée une valeur correspondant à l'une de 3 
classes préalablement définies, en fonction de la durée de submersion et de la fréquence à l'inondation (inondations par crues torrentielles, inondations par un cours d'eau de plaine, inondations par ruissellement urbain, inondations par refoulement du réseau d'assainissement, inondations par remontée de nappe, submersions marines lors de grandes surcotes). Le résultat cartographique a pris en considération les ouvrages de protection (digues et remblais) et les équipements (routes, voies ferrées, ponts...) qui peuvent avoir un impact sur les conditions de submersion et de débordement. Cette méthode est soumise à une certaine subjectivité qui réside dans le choix de la typologie et dans l'interprétation des extraits de Google Earth, de l'assemblage des photographies aériennes et des évènements pluviométriques retenus (2003, 2007 et 2011); (Fig. 7).

Mais aussi, des pluies d'intensité forte, moyenne et parfois faible, mais prolongées peuvent être à l'origine d'une remontée lente des eaux des oueds, du plan d'eau de la sebkha et de la nappe phréatique.

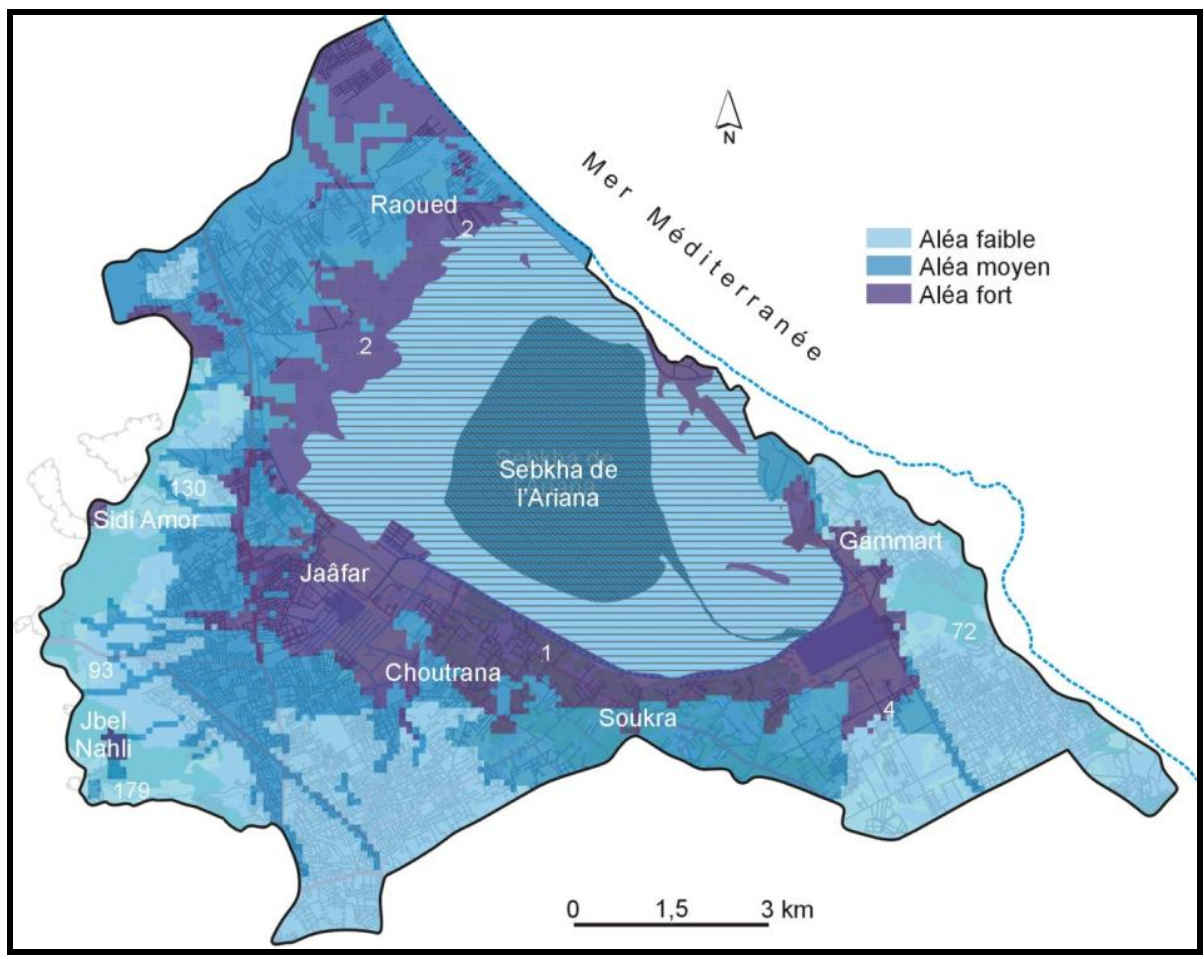

Fig. (7): Quantifications des aléas en fonction de la durée de submersion et de la fréquence à l'inondation. 
Le suivi des évènements pluviométriques des trente dernières années fait ressortir le bassin de l'oued Ennkhilet et les zones basses entourant la sebkha de l'Ariana comme la zone la plus touchée par l'aléa inondation. La carte obtenue montre que les alentours de la sebkha ainsi que la plaine de l'Ariana Nord sont soumis au débordement et à l'engorgement par les eaux au cours des évènements retenus. La configuration de la zone soumise à un aléa fort montre aussi l'impact des aménagements de franchissement du cours d'eau (pont) sur la fréquence d'inondation. Un étalement de la zone à aléa fort est enregistré souvent juste en amont des ouvrages. La limite en ligne droite continue de la zone inondée reflète un détournement des eaux par des remblais opaques hydrauliquement des routes et un dérèglement de la circulation transversale et longitudinale des eaux de débordement dans la plaine alluviale. La plupart des habitations situées dans la plaine de Choutrana et près de Henchir Jaâfar ont été complètement isolées en 2003, 2007 et 2011. Plusieurs habitants confirment devoir faire face à des problèmes liés à l'eau dès qu'il pleut (Chouari, 2009; Fehri et Mathlouthi, 2012). Les inondations dans cette dernière zone ne sont qu'une conjonction de trois types : une inondation par remontée de la nappe, par avancée de la sebkha lors de la saison pluvieuse et par ruissellement de l'amont.

Les fuites dans le réseau de l'ONAS, de stations d'épuration et de canaux associés sont des sources majeures auxquelles s'ajoutent le déversement des grandes quantités d'eau usées par l'intermédiaire des fosses septiques, les excès d'eau d'irrigation (essentiellement des eaux usées traitées pour l'irrigation), l'arrêt durable des pompages importants dans la nappe phréatique, ont provoqué, sur le pourtour de la sebkha, une remontée sensible du niveau d'eau (Photos 3 et 4). Si certains secteurs sont épargnés du débordement direct des oueds, les remontées des nappes qui accompagnent les stagnations des eaux élargissent les zones exposées au risque d'inondation.

Quant aux zones d'épandage ou celles caractérisées par des pentes très faibles et un drainage difficile sont soumis à un aléa moyen. Les eaux circulent donc rapidement sur les versants mais lentement dans la partie urbaine plane et se jettent finalement dans la sebkha tout en causant une situation dangereuse. Cette variation de vitesse des crues de l'amont vers l'aval, peut former un reflux et mène ainsi à des zones très étendues d'épandage, avec des problèmes réels de dépôts solides dans les routes. La zone d'épandage est délimitée par le passage de la route RV533. L'épandage n'est pas continu ; il s'interrompt souvent suite au passage d'une route ou par effets directs ou indirects d'une urbanisation spontanée disposée perpendiculairement au sens d'écoulement.

Les zones en pentes fortes, qui correspond à l'impluvium, ou relativement éloignées de la sebkha sont soumises à un aléa faible. 


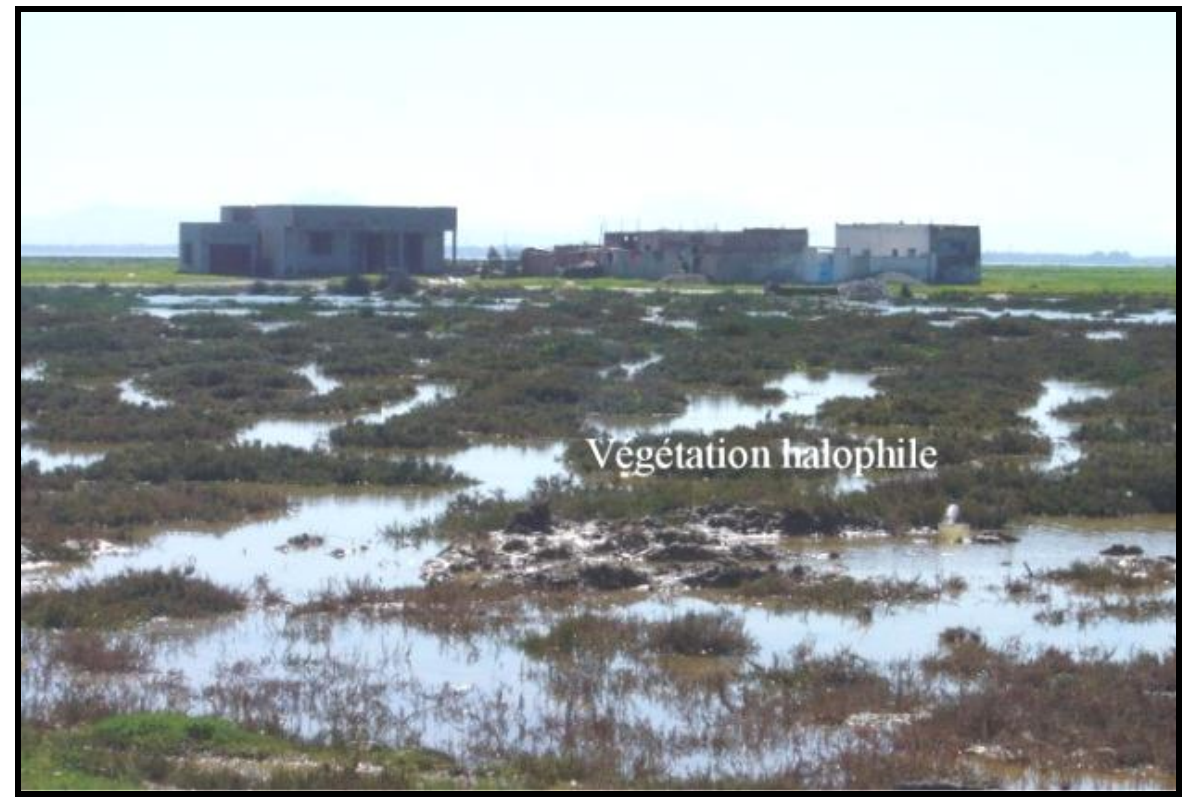

Photo (3): Des nouvelles constructions aux dépens des terres de type sebkha constamment humides au Nord de la sebkha Ariana (W. Chouari, février 2006).

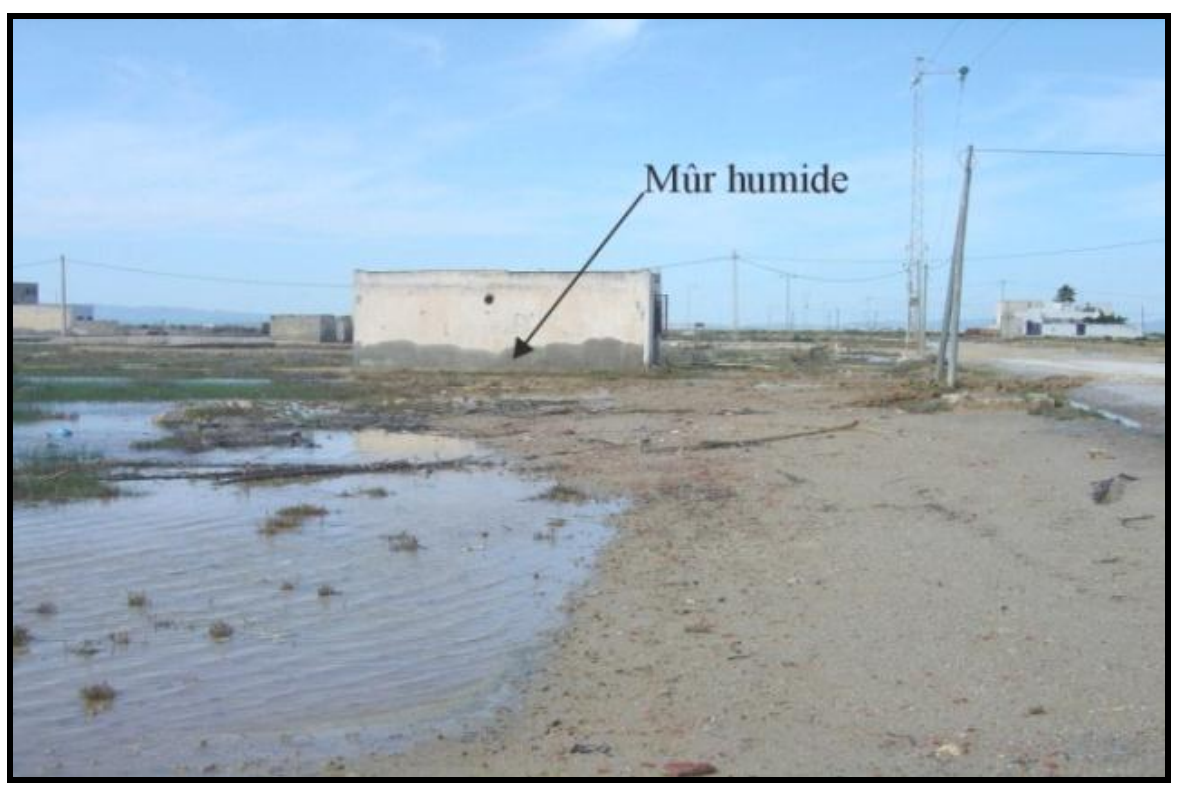

Photo (4): Au Nord de Raoued, des constructions menacées par l'humidité et installées sur un terrain gorgé d'eau plusieurs mois par an (W. Chouari, février 2006). 
Nous visons effectuer aussi un zonage de vulnérabilité hiérarchisant les niveaux d'exposition et de relever les indications relatives aux dommages.

\section{L'évaluation de la vulnérabilité}

Nous essayerons de démontrer comment cette extension urbaine a-t-elle exacerbé la vulnérabilité du milieu y aggravant ainsi le risque hydrologique. Ainsi, dans la cartographie de la vulnérabilité nous avons combiné les classes des pentes et les classes des enjeux, dans une logique qui consiste à dégager le rôle de la pente qui dirige les eaux de pluies. La densité résidentielle est souvent considérée comme le facteur de vulnérabilité le plus évident. La vulnérabilité très faible caractérise les terrains ayant un enjeu très faible et situé sur des pentes fortes. La vulnérabilité moyenne est le résultat d'un enjeu faible et d'une pente très faible ou d'un enjeu fort et d'une pente forte. Une vulnérabilité forte est issue d'un enjeu fort et d'une pente très faible.

Le bilan et l'analyse des dernières inondations (2003, 2007 et 2011) montrent une augmentation préoccupante de la vulnérabilité qui résulte de plusieurs facteurs:

a. L'urbanisation et l'implantation d'activités humaines dans les zones inondables: l'urbanisation récente et l'expansion spatiale de la ville s'est faite en grande partie dans des secteurs attractifs d'un point de vue de l'accessibilité (proches des axes du transport) et économique (nouveau quartier de service) à l'image de la cité El Ghazala et Jâafar, mais, bas souvent sans conscience de leur vulnérabilité (spéculation foncière et terrains à faible prix). En parallèle, l'augmentation du niveau de vie et le développement des réseaux d'infrastructures (transports, énergie, télécommunication, eau...) ont accru dans des proportions importantes la valeur globale des biens et la fragilité des activités exposées;

b. La diminution des champs d'expansion des crues, consécutive à la consommation d'espaces par une agriculture intensive et une urbanisation galopante. Les infrastructures de transports sont des facteurs aggravants puisqu'elles ont été accompagnées de l'édification de digues ou de remblais (levées en terre, remblais de routes et de chemin de fer, ponts...). Ces aménagements ont réduit l'effet naturel d'écrêtement et ont contribué à la concentration et à la stagnation des eaux dans des zones préférentielles.

c. Sur les voies des écoulements (oued Ennkhilet, Tabeuk...), les débâcles et les ruptures de digues s'avèrent particulièrement fatales. Une grande partie de l'écoulement avec sa charge des matériaux solides peut alors se déverser brusquement sur les zones avoisinantes et chercher de nouvelles voies d'écoulement, menaçant ainsi de façon imprévisible des zones moins sensibles situées loin du point de rupture. 
La carte $n^{\circ} 8$ définit trois degrés de vulnérabilité exprimés en trois zones dont l'intensité de la couleur exprime la valeur. Le degré de vulnérabilité varie de 1 (vulnérabilité faible) à 3 (vulnérabilité forte) selon les types d'occupation des sols et les classes des enjeux. La répartition surfacique des classes de vulnérabilité montre la prédominance de la classe des vulnérabilités moyennes, couvrant une superficie de l'ordre de $61 \%$ du bassin versant (Fig. 8).

Les quartiers de 1'Ariana Nord, de Jâafar, de Choutrana et de Sidi Slimane montrent la vulnérabilité la plus forte. Ces quartiers s'étendent sur des terrains très plats et présentent une densité de population assez importante. Une deuxième classe est qualifiée de vulnérabilité moyenne et concerne les cités de Raoued, Sidi Amor et Soukra. La vulnérabilité moyenne s'explique par une densité urbaine moyenne à faible et des pentes très faibles ou des pentes relativement fortes et des densités urbaines moyennes. La classe de vulnérabilité faible intéresse le reste de l'espace à dominance de céréaliculture et à versants à pentes fortes c'est-à-dire à enjeux relativement faibles.

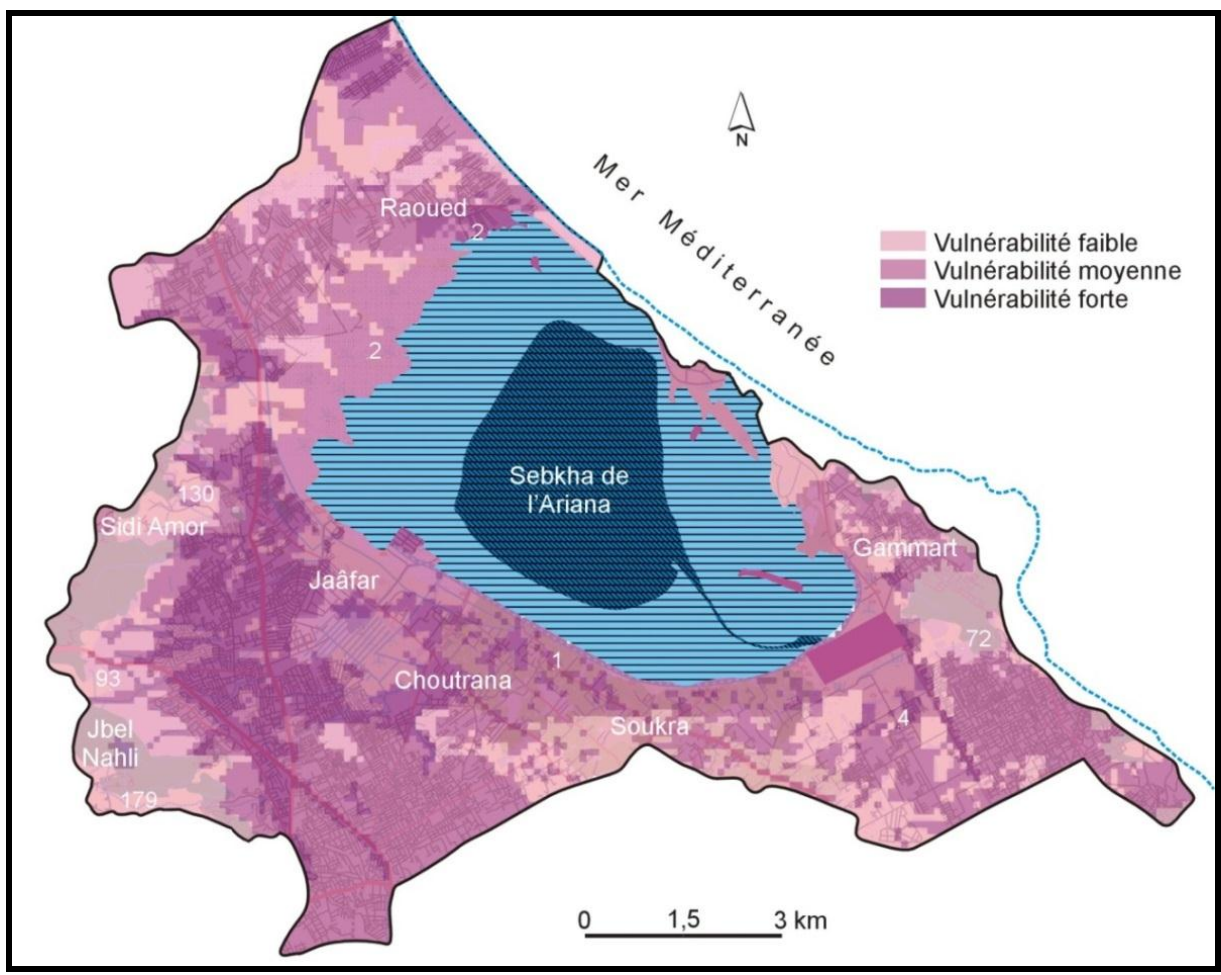

Fig. (8): Carte de vulnérabilité aux inondations dans le bassin versant de la sebkha de l'Ariana. 


\section{La cartographie du risque d'inondation}

Le résultat de la combinaison de la carte des aléas et de la carte des vulnérabilités est une carte des risques d'inondation à trois niveaux:

a. Des zones à risque faible: elles englobent les parties accidentées du bassin versant. Elles correspondent à des terrains naturels nus ou boisés de fortes pentes. Le risque est faible vu les faibles densités urbaines. En outre, dans les zones d'habitat dense et ancien notamment dans le centre de ville de l'Ariana, lorsque des quantités importantes de pluie tombent, les canalisations se révèlent bien souvent incapables d'acheminer les apports d'eaux supplémentaires induits par les ruissellements sur les surfaces imperméabilisées raccordées aux réseaux. Ces refoulements qui surprennent par leur brutalité et leur puissance, ont présenté des risques pour les personnes (projection de plaques d'égouts, invasion des parkings souterrains). Ici le problème est d'ordre technique, le calibrage du réseau a été sous-estimé et l'extension du bâti aux dépens des pentes a amplifié les risques. L'emploi des canalisations étroites est toujours chose courante. La situation sera plus compliquée surtout que certains bassins d'écrêtement et collecteurs d'eau pluviale ne bénéficient pas souvent de l'entretien nécessaire ou n'ont pas toujours été en mesure de contenir les eaux de fortes pluies.

b. Des zones à risque moyen: elles englobent surtout une partie des zones d'épandage où l'occupation anthropique du milieu est encore faible, des interfluves entre les talwegs et des parties des rives de la sebkha de l'Ariana considérées comme des terrains naturels.

Dans la plaine Nord de la sebkha, dépourvue de réseau hydrographique naturel et fortement imperméabilisée, l'inondation par ruissellement pluvial urbain pose beaucoup de problèmes. Des inondations sont possibles aussi après de fortes pluies, liées à la remontée de la nappe phréatique. Sur les rives sud-est de la sebkha, la surface inondée en crue centennale (2003) est d'environ 600 ha. Elle ne concerne que quelques quartiers d'urbanisation récente, moyennement dense, à El Bahr Lazrag et au Douar Essebkha. Notons que la plage de Raoued et les installations touristiques qui s'y trouvent sont également menacées par des submersions marines lors des surcôtes exceptionnelles. Dans le secteur sud-ouest de la sebkha, les problématiques majeures d'inondation se localisent dans le secteur mixte résidentiel et d'activités en bordure de l'aéroport (nouveaux quartiers de Chotrana 2, La Soukra, Sidi Salah et Sidi Zid); (Fig. 9). 


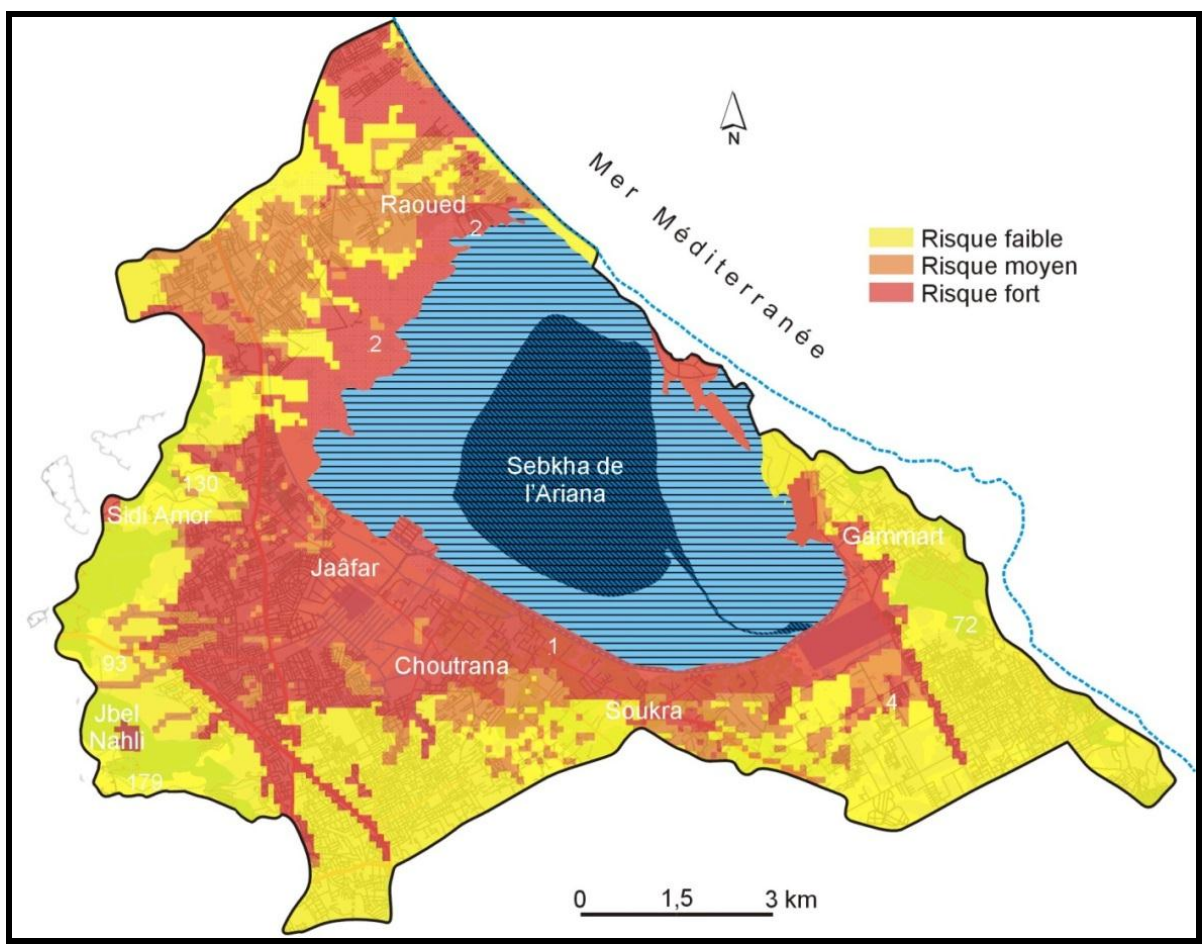

Fig. (9): La carte des risques d'inondations dans le bassin versant de la sebkha de l'Ariana

c. Des zones à risques fort: elles correspondent aux zones de rupture de pente et à la partie centrale de la plaine située entre les routes RV533, RV543 et l'autoroute Tunis-Bizerte, les trois grandes artères de communication. Leur tracé NO-SE est obséquent par rapport à tous les oueds qui drainent le versant est de Jbel Nahli. Ils sont alors à l'origine de débordements répétés. Multiples sont les quartiers à fort risque d'inondation de part et d'autre de ces artères. Tout d'abord le long de la route RV533 surélevée par rapport au talweg de l'oued. La station d'épuration de Choutrana et en pleine zone inondable (Photo 5).

La cité Borj Mâala est située en zone basse où déborde souvent un canal d'évacuation des eaux pluviales provoquant des inondations fréquentes. Henchir Jâafar correspond à une zone d'épandage avec une interruption de l'écoulement par la route RV543. Les routes et les canaux de l'ONAS, «E 4» et «Khélij», constituent de véritables barrages aux écoulements superficiels. Ils modifient le drainage naturel parfois sur de très grandes longueurs, forçant l'écoulement des eaux accumulées vers des passages obligés, généralement placés sur des cheminements naturels apparents (lits d'oued, talwegs...). 

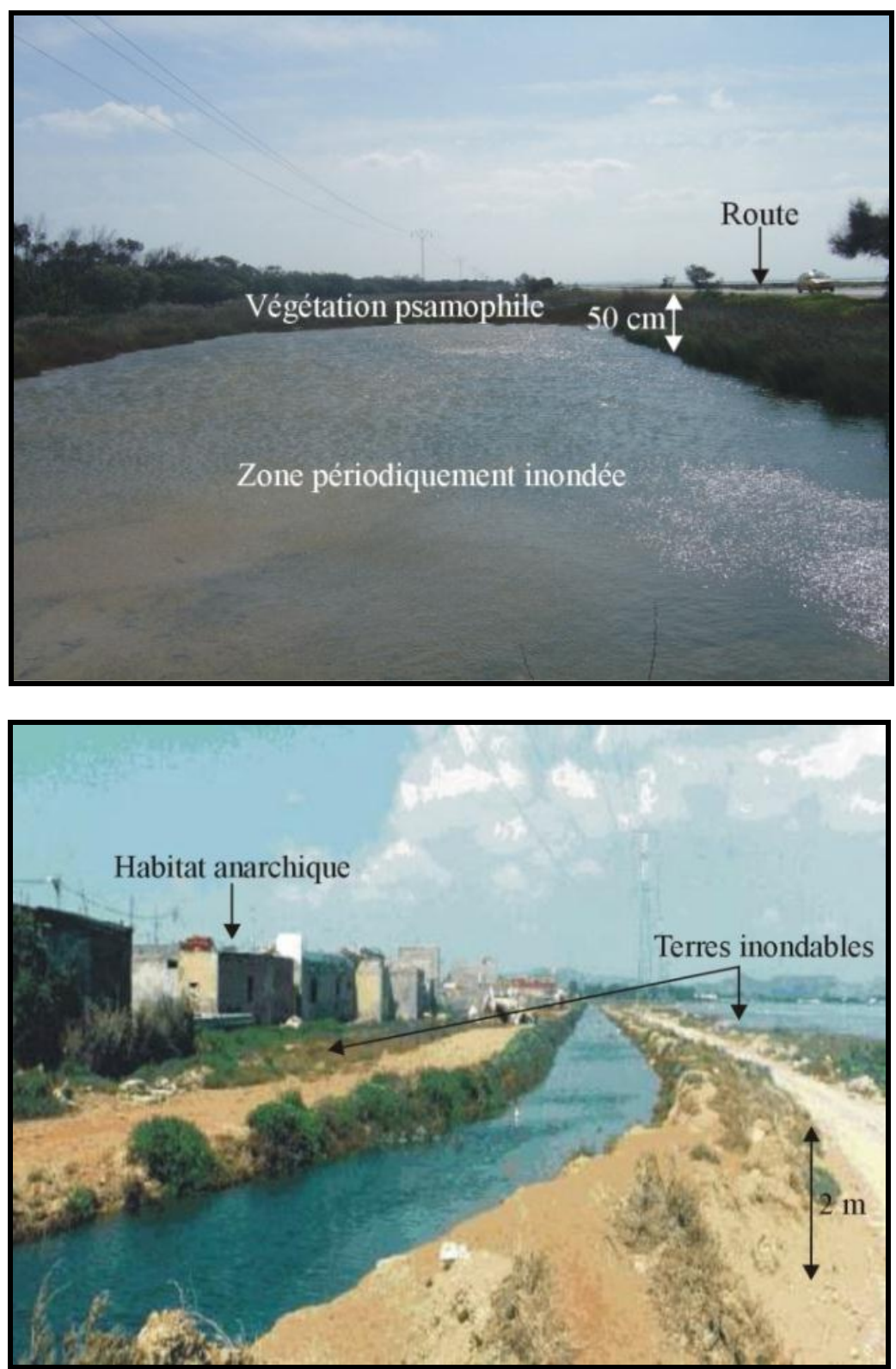

Photo (5): Parmi les obstacles majeurs contre le ruissellement en surface, en direction de la sebkha de l'Ariana, la route de Gammart vers Raoued et le canal des eaux usées qui part de la station d'épuration «Côtière Nord» en direction de la station d'épuration de Choutrana. Les constructions ainsi que les routes surélevées récemment, limitent le ruissellement et génèrent des zones locales d'eaux stagnantes (W. Chouari, été 2006). 
La nette densification du bâti tout au long de la route RV533 s'oppose aux îlots urbains entre lesquels s'intercalent des exploitations agricoles souvent délaissées ou carrément des zones marécageuses appartenant à la station d'épuration de Choutrana. En fait, la majorité des constructions sont anarchiques et disposées selon une direction Nord-Sud faisant obstacle aux écoulements. La voirie, si elle existe, est non aménagée ou revêtue et non accompagnée de réseau de drainage pluvial et même du réseau d'assainissement des eaux usées. Prenons le cas de la plaine de Choutrana, drainée par trois principaux canaux d'évacuation d'eau pluviale, lors de notre visite du terrain nous avons remarqué que:

a. Les bassins d'écrêtement réalisés sur le canal Nord qui ceinture la ville de l'Ariana le long de la route de Bizerte puis traverse à partir du bassin d'écrêtement la plaine de Choutrana par la cité Ezzahani et Sidi Zid vers la sebkha, sont mal entretenus et sont envasés;

b. Le canal Ouest, qui longe la route RVE533 (route de Raoued) entre l'autoroute et Henchir Jâafar pour se jeter dans la sebkha par Sidi Sliman traverse une zone urbaine plus ou moins dense et en extension rapide et se trouve occupé par la végétation et les rejets d'ordures;

c. Les différents canaux réalisés en 1985 dans le périmètre irrigué de Soukra et Choutrana, n'étaient pas entretenus. Ils ne remplissent plus actuellement leur fonction, d'autant plus que leurs rejets vers la sebkha assurés par siphonage au niveau du canal ONAS se trouvent complètement bouchés.

L'oued Ennkhilet affecte des terrains assez étendus. On estime que les zones résidentielles représentent près du $1 / 3$ des aires directement affectées par les crues de cet oued et la surface inondable concerne principalement des zones d'habitation moyennement denses. Les inondations sont considérées par les résidents comme des phénomènes temporaires et, par conséquent, comme nuisance. Les risques d'inondations concernent essentiellement des nouveaux secteurs d'habitation (construits dans les 25 dernières années) qui, semble-t-il, n'ont pas été conçus en intégrant pleinement ces risques et contribuent même à les augmenter.

À l'Ariana Nord, les risques humains et économiques sont considérables en raison de nombreux facteurs aggravants: concentration des personnes, des activités et des biens et la formation courante d'une succession d'embâcles et de débâcles. Ces risques pour la vie des personnes et l'intégrité des biens sont d'autant plus élevés qu'un important charriage de matériaux rend souvent les flots plus destructeurs. Là encore l'avertissement de la population est très difficile. Les inondations de septembre 2003 et d'octobre 2007, qui ont ébranlé l'agglomération tunisoise représentent bien ce type d'inondation. 
En septembre 2003, au pic de l'inondation, une immense lame d'eau atteignait et recouvrait une hauteur moyenne de $0,5 \mathrm{~m}$, la quasi-totalité de la plaine du sous-bassin versant d'Ennkhilet (Chouari, 2009). Cette lame d'eau recouvrait et recouvre encore épisodiquement sans distinction, zones urbaines, agricoles et naturelles. La partie aval de l'oued Ennkhilet subit souvent une augmentation des débits sous l'effet cumulatif des différents tronçons du réseau d'où des phénomènes de débordements fréquents. En outre, du fait de la faible taille du sous-bassin versant, le temps de réponse du cours d'eau est rapide et la pointe de crue se cale presque sur le pic d'intensité pluviométrique. Les dernières inondations (2003, 2007 et 2011) ont montré que les crues se heurtent facilement à la sous-capacité du réseau d'évacuation des eaux pluviales et à la désorganisation du réseau hydrographique naturel. L'eau emprunte souvent les rues qui se transforment en torrent. En plus, plusieurs obstacles ont été implantés dans les cours naturels des oueds et dont les effets sont perceptibles surtout en aval (sur les rivages de la sebkha) où s'étendent des terres hydromorphes et où l'eau trouve les conditions topographiques et hydrologiques favorables pour stagner et séjourner pendant plusieurs mois de l'année. Les problématiques majeures d'inondation se localisent dans la zone nord de l'agglomération d'Ariana: cités Ettaami, Al Baladia, El Mostakbel, Essaad. La surface inondée en crue centennale (2003) est d'environ 800 ha.

En s'approchant de la sebkha le secteur est soumis à des phénomènes d'inondations pluviales, mais aussi indirectement de submersion via la sebkha. Le risque de submersion marine pour une tempête exceptionnelle concerne essentiellement les zones naturelles bordant la sebkha et la zone marécageuse s'étendant entre l'École des PTT (Postes, Télégraphes et Téléphones) et Sidi Slimane. Le niveau d'eau dans la Sebkha de l'Ariana conditionne le bon drainage de ce secteur où des inondations se produisent même pour des pluies relativement fréquentes, puisque le réseau pluvial actuel ne peut écouler que $51 \%$ du débit décennal (Ennesser et al., 2011). Soumises à une urbanisation anarchique qui créée des obstacles aux écoulements et à des phénomènes de remontée de nappe, ces zones basses connaissent de fréquentes et longues périodes d'inondation. Cette situation est d'autant plus inquiétante qu'au cours des dernières années, la nappe phréatique du secteur connait une remarquable surélévation de son niveau piézométrique suite aux rejets d'eaux usées dans des fosses septiques par des habitations qui ne sont pas connectées au réseau d'assainissement et les rejets d'eau à partir de la station d'épuration de Choutrana dans la sebkha (Chouari, 2009 et 2015 ; Fehri et Mathlouthi, 2012).

Dans la zone de Soukra-Choutrana, un inventaire de puits a été réalisé par le CRDA (Commissariat Régionale de développement Agricole) d'Ariana 
en 1973. Cet inventaire fait ressortir 1558 puits dont 264 sont abandonnés (Chouari, 2009). Nos investigations de terrain ont montré l'augmentation du nombre de puits nouvellement abandonnés. Ceci implique une diminution du débit d'exploitation des eaux de la nappe phréatique induisant sa remontée. Le réseau de surveillance, contrôlé par le CRDA, met en évidence une remontée appréciable du niveau statique de la nappe libre de Soukra dont les manifestations sont sommairement représentées par les nivellements de cinq puits représentés dans la figure ci-dessous (Fig. 10).

La remontée moyenne a été estimée de 1,57 m entre 1975 et 1997 soit 7 $\mathrm{cm} / \mathrm{an}$. Les nouvelles mesures estimées au niveau de la nappe de SoukraChoutrana ont montré que celle-ci est largement sous-exploitée. L'exploitation en 1997 est de l'ordre de 5,9 $106 \mathrm{~m}^{3} / \mathrm{an}$, alors que les ressources exploitables sont de l'ordre de $10106 \mathrm{~m}^{3} /$ an en 1975 (Sfar-Falfoul, 1998).

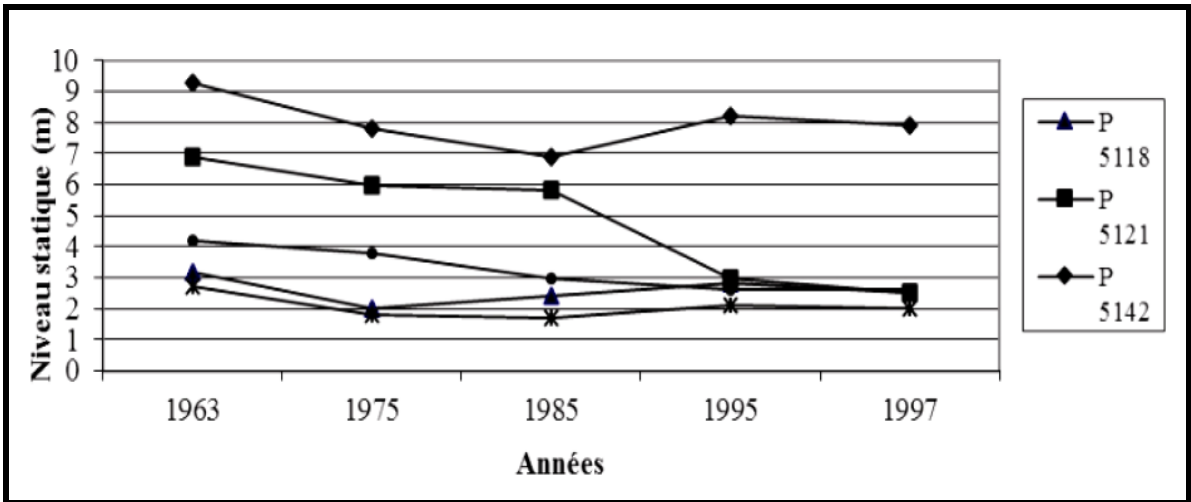

Fig. (10): Courbes des fluctuations des niveaux statiques de cinq puits de la plaine de Soukra entre 1963 et 1997.

Par ailleurs, ces inondations lentes présentent peu de risques pour les personnes, mais provoquent des dommages non négligeables à la voirie et aux constructions. Les affleurements d'eau souterraine ont engendré la perte de grandes parcelles plantées d'oliviers séculaires. Elle a contribué également à détruire des hectares d'agrumes dans la région de Soukra. Dans ce dernier secteur, il est fréquent de voir des routes, et terrains agricoles plats (à dominance de culture maraîchère et annuelle) s'éparpillant le long des rives de la sebkha de l'Ariana inondées de plusieurs centimètres d'eau de pluies à cause de l'insuffisance du réseau de drainage des eaux pluviales. La remontée de la nappe atteint aussi les sous-sols et les ouvrages enterrés (canalisations de l'ONAS et canalisation de drainage agricole) et parfois la surface du sol. Elle rend ces ouvrages inutilisables sur le moment ou en permanence. 
Nous sommes en présence d'un bilan hydrologique perturbé par une occupation humaine spontanée et progressive. L'imperméabilisation des sous-bassins versants amont a augmenté les apports en eaux de ruissellement, alors que l'urbanisation anarchique des quartiers spontanés de la plaine souvent perpendiculaires aux lignes des talwegs, entrave les écoulements et provoque le comblement des fossés de drainage.

Le réseau d'évacuation des eaux pluviales, est soit sous-dimensionné ou très général avec une absence de réseaux secondaires de captage provoquant ainsi l'inondation, surtout de la zone aval, pour des pluies de fréquence supérieure à la fréquence annuelle. Il s'agit de tout un système complexe et il faut travailler conjointement sur l'état des nappes, sur l'apport des cours d'eau et sur l'apport issu des zones urbanisées, car tous ces facteurs se conjuguent pour produire l'inondation.

\section{Conclusion}

Dans ce milieu naturellement fragile, l'ampleur des phénomènes météorologiques, topographiques, géologiques, hydrogéologiques et biogéographiques a été renforcée par les modalités actuelles de l'occupation du sol et de gestion des lits des oueds. Jusqu'à une période récente, le comportement des nappes et le fonctionnement hydrologique de l'ensemble de l'écosystème suivait un cycle régulier, caractérisé par un assèchement complet de la sebkha durant l'été, favorisant ainsi le drainage des nappes. En hiver, les nappes se rechargeaient à travers les formations perméables des versants environnants et les sols de texture grossière de la plaine de Soukra (dune de Soukra). Cet équilibre n'était que rarement perturbé, même durant les années de pluies exceptionnelles.

Aujourd'hui, pour les petits cours d'eau intermittents avoisinants et traversant la zone urbaine, la spécificité du risque d'inondation ne réside donc pas dans la nature de l'aléa mais surtout dans le régime contrasté des pluies, le mode d'aménagement de la section et la période de retour prise en considération et surtout le sentiment d'oubli de la population à l'égard des crues. Ces spécificités se doublent par un bassin versant à relief qui favorise le ruissellement et la concentration rapide des eaux dans les zones basses, de la morphologie des voies de communication «en rateau» qui restreint les échappatoires. Il est évident aussi de l'analyse de la relation existant entre les inondations et les événements pluvieux que les pluies responsables des inondations de ces dernières années ne revêtent pas obligatoirement un caractère exceptionnel. La vulnérabilité, notamment du bassin versant de la sebkha de l'Ariana, face au risque d'inondation semble avoir fortement augmenté suite à un aménagement urbain déficient. La fréquence d'inondation 
est devenue telle que toute activité économique se trouve perturbée et ceci à la moindre averse.

Face à la pression anthropique sur le milieu, une volonté de l'État se dessine en faveur de la protection de cet espace fragile. Toutefois les obstacles à cette nouvelle gestion conservatoire sont nombreux et les progrès sont très lents. Les moyens techniques de protection contre les inondations (digues, bassins d'écrêtement,...) peuvent uniquement améliorer les conditions des usages en bordure des cours d'eau, et non pas, écarter totalement le risque d'inondation en soi. Car les crues exceptionnelles entraînent à nouveau des inondations dans des zones considérées comme protégées. Ainsi, la gestion du risque, historiquement fondée sur le contrôle et la limitation des aléas, s'est tournée vers une tentative de réduction de la vulnérabilité des enjeux. Et, dans cette étude, les propositions méthodologiques de cartographie visent donc à pallier certaines faiblesses de travaux antérieurs, et invitent à agir désormais sur les vulnérabilités de manière concertée, impliquant, à travers une démarche participative, tous les acteurs du territoire. Par ailleurs, aussi cette approche n'est pas sans certaines limites, qu'il convient de prendre en compte.

\section{Bibliographie}

- Baltas E.A., Dervos N.A., et Mimikou M.A. (2007), Technical Note: Determination of the SCS initial abstraction ratio in an experimental watershed in Greece. Hydrology and Earth System Sciences, vol. 11, $\mathrm{n}^{\circ}$ 6, pp. 1825-1829. DOI: $10.5194 /$ hess-11-1825-2007

- Chouari W., 2003, Les dépressions humides des environs de Tunis: géomorphologie, paléoenvironnement et impact des aménagements sur leur évolution. Mémoire de DEA, Université Paris I, 116 p.

- Chouari W., 2006, Les pluies torrentielles des 17 et 24 septembre 2003 dans le Grand Tunis : analyse météorologique et impacts immédiats. In : Les risques liés au temps et au climat, XIX ${ }^{\text {ème }}$ colloque de l'AIC (Épernay), G. BELTRANDO, M. MADELIN et H. QUÉNOL édit., Édit. PRODIG, Paris, pp. 166-171.

- Chouari W., 2009, Environnement et risques naturels dans le Grand Tunis, approche cartographique. Thèse de l'Université Paris VII, 408 p.

- Chouari W., 2015, Occupation des sols et morphodynamique récente dans le bassin versant de la sebkha de l'Ariana (Tunisie Nord-orientale), Confins [Online], 24 | 2015.

- DGAT - GRAPHTECH, 2011, Atlas du gouvernorat de l'Ariana, Tunis, 88 p.

- Dlala H., 2007, «Métropolisation et recomposition territoriale du Nord-Est tunisien», Cybergeo: European Journal of Geography [En ligne], Environnement, Nature, Paysage, 2007, URL: http://cybergeo.revues.org/13863, $\mathrm{N}^{\circ} 410,3$ décembre, 2007,

- Fehri N. et Mathlouthi S., 2012, «Extension urbaine et risque d'inondation sur le piémont est du Jbel Ennahli (Aiana-Tunisie) », in Actes du VIIème Colloque du Département de Géographie. De la Manouba, 6-8 Mars 2008, 14 p. 
- Fehri N. et Zahar Y., 2016, «Étude de l'impact de l'extension et de la densification du tissu urbain sur les coefficients de ruissellement dans le bassin versant des oueds El-Ghrich et El-Greb (Tunis) par l'application de la méthode SCS aux évènements de septembre 2003 », Physio-Géo, 10(1): 61-79.

- Ennesser Y., Said V. et Terrier M., 2011, Évaluation des risques en situation actuelle et à l'horizon 2030 pour la ville de Tunis. Rapport final de Phase $1 \mathrm{du}$ Projet "Adaptation au changement climatique et aux désastres naturels des villes côtières de l'Afrique du Nord, Egis BCEOM/IAU-IDT/BRGM, 342 p.

- URL: http://www.iau-idf.fr/fileadmin/Etudes/etude_823/Tunis_Rapport_Phase_1.pdf.

- JICA, 1994, Étude sur le programme de protection contre les inondations pour le Grand Tunis et le Grand Sousse. Japan International Cooperation Agency, rapport final au Ministère de l'Équipement et de l'Habitat, Tunis, 376 p.

- Mishra S.K. et Singh V.P., 2003, Soil conservation service curve number (SCSCN) methodology. Édit. SPRINGER, collection "Water sciences and technology", Vol. 42, Dordrecht (Pays-Bas), XX + 456 p. +.annexes. DOI: $\underline{10.1007 / 978-94-017-0147-1}$

- Mockus V., 1972, Estimation of direct runoff from storm rainfall. (SCS). In: National engineering handbook, section 4: Hydrology, chapitre 10, III + 24 p.

- Nasralla, W., 2013, Impacts de l'urbanisation et des aménagements sur le ruissellement et les écoulements dans la banlieue sud du Grand Tunis : cas des bassins-versants exoréiques entre Hammam-Lif et Borj Cédria., Mém. de Mastère. Faculté des Lettres, des Arts et Humanités de la Manouba, 99 p.

- Oueslati A., 1999, Les inondations en Tunisie, Orbis, Tunis, 206 p.

- Sfar-Falfoul F., 1998, Étude de l'évolution naturelle du système actuel de la Sebkha d'Ariana: Apport de la télédétection et des systèmes d'informations géographiques, mémoire de D.E.A. Faculté des sciences de Tunis, 123 p.

- SWECO-APAL, 2000, Étude de l'assainissement de la sebkha de l'Ariana, A.P.A.L., Tunis, $171 \mathrm{p}$.

- Zarai F., 2000, Urbanisation illégale et milieu naturel dans la banlieue de l'Ariana, In «Connaissance et pratiques des milieux et territoires», 3ème colloque du Département de Géographie de la Faculté des Sciences Humaines et Sociales de Tunis, Publications de l'École Normale Supérieure de Tunis, 15-35 (en langue arabe).

- Zarai F., 2006, L'extension urbaine dans l'une des banlieues de Tunis: cas de l'Ariana Nord. (En arabe). Publications de l'Université de Tunis, 370 p. 